\title{
A PROOF OF THE MOD 4 UNIMODAL SEQUENCE CONJECTURES AND RELATED MOCK THETA FUNCTIONS
}

\author{
RONG CHEN AND F. G. GARVAN \\ Dedicated to the memory of Freeman Dyson
}

\begin{abstract}
In 2012 Bryson, Ono, Pitman and Rhoades showed how the generating functions for certain strongly unimodal sequences are related to quantum modular and mock modular forms. They proved some parity results and conjectured some mod 4 congruences for the coefficients of these generating functions. In $2016 \mathrm{Kim}$, Lim and Lovejoy obtained similar results for odd-balanced unimodal sequences and made similar mod 4 conjectures. We prove all of these mod 4 conjectures and similar congruences for the Andrews sptfunction and related mock theta functions. Our method of proof involves new Hecke-Rogers type identities for indefinite binary quadratic forms and the Hurwitz class number.
\end{abstract}

\section{INTRODUCTION}

We prove some conjectured congruences mod 4 for certain unimodal sequences. We follow Bryson, Ono, Pitman and Rhoades's [18] definition of a strongly unimodal sequence. A sequence of integers $\left\{a_{j}\right\}_{j=1}^{s}$ is a strongly unimodal sequence of size $n$ if it satisfies

$$
0<a_{1}<a_{2}<\cdots<a_{k}>a_{k+1}>\cdots>a_{s}>0 \text { and } a_{1}+a_{2}+\cdots+a_{s}=n,
$$

for some $k$. Let $u(n)$ be the number of such sequences, and define the rank of such a sequence as $s-2 k+1$; i.e. the number terms after the maximum minus the number of terms before it. Let $u(m, n)$ be the number of strongly unimodal sequences of size $n$ and rank $m$. Then

$$
\mathcal{U}(z ; q):=\sum_{m, n} u(m, n) z^{m} q^{n}=\sum_{n=0}^{\infty}(-z q ; q)_{n}\left(-z^{-1} q ; q\right)_{n} q^{n+1},
$$

Date: October 28, 2020.

2020 Mathematics Subject Classification. 05A17, 05A39, 11E41, 11F33, 11F37, 11P81, 11P83, 11P84, $33 \mathrm{D} 15$.

Key words and phrases. Partitions, mock theta functions, Ramanujan-type congruences, Hurwitz class number, unimodal sequences, Andrews spt-function, Hecke-Rogers series.

The first author was supported in part by the National Natural Science Foundation of China (Grant No. 11971173) and an ECNU Short-term Overseas Research Scholarship for Graduate Students (Grant no. 201811280047). The second author was supported in part by a grant from the Simon's Foundation (\#318714). 
where we use the usual q-notation

$$
(a ; q)_{n}:=\prod_{k=0}^{n-1}\left(1-a q^{k}\right) .
$$

In addition, let $u(a, b ; n)$ be the number of strongly unimodal sequences of size $n$ and rank congruent to $a \bmod b$.

Bryson, Ono, Pitman and Rhoades [18] relate $\mathcal{U}(-1 ; q)$ with a quantum modular form which is dual to a quantum modular form of Zagier [44. They also show that $\mathcal{U}( \pm i ; q)$ is mock modular form and prove interesting parity results for the coefficients $u(n)$. They made the following conjecture.

Conjecture 1.1. Suppose $\ell \equiv 7,11,13,17(\bmod 24)$ is prime and $\left(\frac{k}{\ell}\right)=-1$. Then for all $n$ we have

$$
u\left(\ell^{2} n+k l-s(\ell)\right) \equiv 0 \quad(\bmod 4)
$$

where $s(\ell)=\frac{1}{24}\left(\ell^{2}-1\right)$. Moreover, for $a \in\{0,1,2,3\}$ we have

$$
u\left(a, 4 ; \ell^{2} n+k l-s(\ell)\right) \equiv 0 \quad(\bmod 2),
$$

and

$$
u\left(0,4 ; \ell^{2} n+k l-s(\ell)\right) \equiv u\left(2,4 ; \ell^{2} n+k l-s(\ell)\right) \quad(\bmod 4) .
$$

Remark. Here and throughout this paper $\left(\begin{array}{l}\cdot \\ \cdot\end{array}\right)$ denotes the Kronecker symbol.

In this paper we prove Conjecture 1.1 and a similar conjecture for a related unimodal sequence function studied by Kim, Lim and Lovejoy [31. We note that there is a stronger result for 1.4 which include primes congruent to $1, \pm 5(\bmod 24)$. See Theorem 4.15. We also prove analogous mod 4 results for Andrews's [2] spt-function and for the coefficients of related mock theta functions.

We describe Kim, Lim and Lovejoy's odd-balanced unimodal sequence. A sequence of integers $\left\{a_{j}\right\}_{j=1}^{s}$ is unimodal of size $n$ if it satisfies

$0<a_{1} \leq a_{2} \leq \cdots \leq a_{k-1}<a_{k}>a_{k+1} \geq \cdots \geq a_{s-1} \geq a_{s}>0$ and $a_{1}+a_{2}+\cdots+a_{s}=n$,

Such a unimodal sequence is called odd-balanced if the peak $a_{k}$ is even, even parts to the left and right of the peak are distinct and the odd parts to the left of the peak are identical with those to the right. As before the rank is the number to right of the peak minus the number to the left. We let $v(n)$ be the number of odd-balanced unimodal sequences of size $2 n+2$ and let $v(m, n)$ be the number with rank $m$. Then

$$
\mathcal{V}(z ; q):=\sum_{m, n} v(m, n) z^{m} q^{n}=\sum_{n=0}^{\infty} \frac{(-z q ; q)_{n}\left(-z^{-1} q ; q\right)_{n} q^{n}}{\left(q ; q^{2}\right)_{n+1}}
$$


Kim, Lim and Lovejoy obtained quantum modular, mock modular and parity results analogous to Bryson, Ono, Pitman and Rhoades's results. They point out the mock modularity properties of $\mathcal{V}(z ; q)$ follow from some results of Mortenson [36, Theorem 4.4]. They also made the following

Conjecture 1.2. Let $\ell \not \equiv-1(\bmod 8)$ be prime and let $k$ be a positive integer satisfying $\ell \| 8 k+7$. If $(8 k+7) / \ell$ is a quadratic residue modulo $\ell$, then

$$
v\left(\ell^{2} n+k\right) \equiv 0 \quad(\bmod 4)
$$

For example, $v(9 n+4) \equiv v(25 n+11) \equiv v(25 n+16) \equiv 0(\bmod 4)$. Moreover, the same congruences hold for the coefficients of $\mathcal{V}( \pm i, q)$.

Remark. This conjecture is false. For example let $\ell=17$ and $k=99$. Then $(8 k+7) / \ell=47$ is a quadratic residue mod 17 , but

$$
v(99)=81474897186 \equiv 2 \quad(\bmod 4) .
$$

The primes congruent to 1 mod 8 must be excluded. The correct version of this conjecture, which we prove, is given below in Theorem 4.1 .

The Andrews's spt-function [2] is related to strongly unimodal sequences. See equations (1.5) and (1.6) below. Andrews defined $\operatorname{spt}(n)$ as the number of smallest parts in the partitions of $n$, and proved that it satisfied some surprising Ramanujan-type congruences mod 5, 7 and 13. Bringmann [16] showed the spt generating function is related to a weight $3 / 2$ harmonic Maass form. The second author made the following

Conjecture 1.3. 1 Suppose $\ell>3$ is prime and $\ell \not \equiv 23(\bmod 24)$. Let $\tilde{\varepsilon}=\tilde{\varepsilon}(\ell)=1$ if $\ell \equiv 1$ $(\bmod 24)$ and -1 otherwise. Then

$$
\operatorname{spt}(\ell n-s(\ell)) \equiv 0 \quad(\bmod 4), \quad\left(\text { where } s(\ell)=\frac{1}{24}\left(\ell^{2}-1\right)\right)
$$

when $\left(\frac{n}{\ell}\right)=\widetilde{\varepsilon}$.

We prove this conjecture in Section 4.2.

The three conjectures above are related to some mod 4 properties for the coefficients of certain mock theta functions. Mock theta functions were first defined by Ramanujan in his famous last letter to Hardy [39, pp.354-355]. In this letter he gave examples of 17 such functions: four of order 3, ten of order 5 and three of order 7 . Since then more mock theta functions have been found by others. See for example Watson [41], Andrews and Hickerson [9], Gordon and McIntosh [26], Berndt and Chan [13] and McIntosh [33]. Many Ramanujantype congruences for mock theta functions have been found mainly to modulus relatively prime to 6. See for example [10]. In a quite recent paper Wang [42] obtained some extensive parity results for 21 of the 44 classical mock theta functions. In this paper we consider mock

\footnotetext{
${ }^{1}$ This conjecture was presented by the second author in a talk, entitled The Andrews spt-function mod 4 , at the AMS Special Session on Arithmetic Properties of Sequences from Number Theory and Combinatorics, AMS Annual Meeting, Atlanta, January 4, 2017.
} 
theta congruences mod 4 . The mod 4 congruences are much harder to prove. Conjecture 1.1 is related to Ramaujan's third order mock theta functions $\psi(q)$. In fact,

$$
\mathcal{U}( \pm i ; q)=\psi(q)=\sum_{n=1}^{\infty} N_{\psi}(n) q^{n}=\sum_{n=1}^{\infty} \frac{q^{n^{2}}}{\left(q ; q^{2}\right)_{n}} .
$$

The function $\psi(q)$ is also related to the spt-function. Andrews, Liang and the second author [8] showed that

$$
\operatorname{spt}(n) \equiv(-1)^{n-1} N_{\psi}(n) \quad(\bmod 4) .
$$

This means that $N_{\psi}(n)$ also satisfies Conjecture 1.3 .

Conjecture 1.2 is related to the second order mock theta function

$$
A(q)=\sum_{n=0}^{\infty} N_{A}(n) q^{n}=\sum_{n=0}^{\infty} \frac{q^{(n+1)^{2}}\left(-q ; q^{2}\right)_{n}}{\left(q ; q^{2}\right)_{n+1}^{2}}=\sum_{n=0}^{\infty} \frac{q^{n+1}\left(-q^{2} ; q^{2}\right)_{n}}{\left(q ; q^{2}\right)_{n+1}}
$$

In fact,

$$
q \mathcal{V}( \pm i ; q)=A(q)
$$

and Kim, Lim and Lovejoy [31, Conjecture 1.3] made the following conjecture.

Conjecture 1.4. Let $p \not \equiv 7(\bmod 8)$ be an odd prime, suppose $8 \delta_{p} \equiv 1\left(\bmod p^{2}\right)$ and $k, n \in \mathbb{Z}$ where $\left(\frac{k}{p}\right)=1$. Then

$$
N_{A}\left(p^{2} n+(p k+1) \delta_{p}\right) \equiv 0 \quad(\bmod 4) .
$$

In a previous paper [20] we found some weight $3 / 2$ eta-products that satisfy a similar mod 4 behaviour. For example, let

$$
f(q):=\sum_{n=0}^{\infty} a(n) q^{n}:=\frac{J_{3}^{3} J_{2}^{2}}{J_{1}^{2}}
$$

where we have used the common notation

$$
J_{k}:=\left(q^{k} ; q^{k}\right)_{\infty}:=\prod_{n=1}^{\infty}\left(1-q^{k n}\right), \quad \text { and } \quad(z ; q)_{\infty}:=\prod_{n=1}^{\infty}\left(1-z q^{n-1}\right) .
$$

The following is a typical result we found.

Theorem $1.5\left(\left[20\right.\right.$, Theorem 1.3]). Let $p>3$ be prime. Suppose $24 \delta_{p} \equiv 1\left(\bmod p^{2}\right)$, and $k$, $n \in \mathbb{Z}$ where $\left(\frac{k}{p}\right)=1$. Then

$$
a\left(p^{2} n+(p k-11) \delta_{p}\right) \equiv 0 \quad(\bmod 4), \quad \text { if } p \not \equiv 11 \quad(\bmod 24) .
$$


A crucial aspect of the proof involves the connection with ternary quadratic forms and the class number of imaginary quadratic fields. The approach in this paper is similar and involves the Hurwitz class number.

In Section 2 we collect and prove some needed results for the Hurwitz class number function $H(N)$. In Section 3 we study four mock theta functions related to the Hurwitz class number function: $A(q)$ (second order), $\phi_{-}(q)$ and $\sigma(q)$ (sixth order), and $V_{1}(q)$ (eighth order), and prove some surprising congruences mod 4 for their coefficients. In Section 4 we study the third order mock theta function $\psi(q)$, and we prove the main conjectures for strongly unimodal sequences, odd-balanced unimodal sequences and the Andrews spt-function.

In this paper, we will often use the following elementary congruences.

$$
\left\{\begin{array}{c}
\frac{J_{1}^{2}}{J_{2}}=1+2 \sum_{n=1}^{\infty}(-1)^{n} q^{n^{2}} \equiv 1 \quad(\bmod 2), \quad \frac{J_{2}^{5}}{J_{4}^{2} J_{1}^{2}}=1+2 \sum_{n=1}^{\infty} q^{n^{2}} \equiv 1 \quad(\bmod 2) \\
\quad \text { and } \frac{J_{1}^{4}}{J_{2}^{2}} \equiv 1 \quad(\bmod 4)
\end{array}\right.
$$

These follow from the well-known Jacobi triple product identity [3, Theorem 3.4, p.461]:

$$
(z ; q)_{\infty}\left(z^{-1} q ; q\right)_{\infty}(q ; q)_{\infty}=\sum_{n=-\infty}^{\infty}(-1)^{n} z^{n} q^{n(n-1) / 2} .
$$

We will also use the following special cases of 1.10 .

$$
\begin{gathered}
\sum_{n=0}^{\infty} q^{n(n+1) / 2}=\frac{J_{2}^{2}}{J_{1}} \\
\sum_{k=-\infty}^{\infty} q^{k(3 k+1) / 2}=\frac{J_{3}^{2} J_{2}}{J_{6} J_{1}}
\end{gathered}
$$

As well as the triple product identity we will use the quintuple product identity [3, Theorem 3.9, p.467]:

$$
\begin{aligned}
& (-z ; q)_{\infty}\left(-z^{-1} q ; q\right)_{\infty}\left(q z^{2} ; q^{2}\right)_{\infty}\left(z^{-2} q ; q^{2}\right)_{\infty} \\
& \quad=\sum_{n=-\infty}^{\infty}(-1)^{n} z^{3 n} q^{n(3 n-1) / 2}+\sum_{n=-\infty}^{\infty}(-1)^{n} z^{3 n+1} q^{n(3 n+1) / 2}
\end{aligned}
$$

\section{The Hurwitz Class number}

Following [22, Section 5.3] we define the Hurwitz class number $H(N)$, where $N$ is a nonnegative integer, as follows.

(1) If $N \equiv 1,2(\bmod 4)$ then $H(N)=0$.

(2) If $N=0$ then $H(0)=-1 / 12$. 
(3) If $N>0, N \equiv 0,3(\bmod 4)$, then $H(N)$ is the class number of positive definite binary quadratic forms of discriminant $-N$, with those classes that contain a multiple of $x^{2}+y^{2}$ or $x^{2}+x y+y^{2}$ counted with weight $1 / 2$ or $1 / 3$, respectively.

It is known that (see for example [43])

$$
H(n)=\frac{2 h(D)}{\omega(D)} \sum_{d \mid f} \mu(d)\left(\frac{D}{d}\right) \sigma_{1}(f / d),
$$

if $n=-D f^{2}$, where $-D$ is a fundamental discriminant, $h(D)$ is the class number of $\mathbb{Q}(\sqrt{D})$, $\mu$ is the Möbius function, $\sigma_{1}$ is the divisor sum, and $\omega(D)$ is the number of units in the ring of integers of $\mathbb{Q}(\sqrt{D})$. As mentioned before $\left(\begin{array}{l}\cdot \\ \cdot\end{array}\right)$ is the Kronecker symbol. In particular for $f=1$ we have

$$
H(-D)=\frac{2 h(D)}{\omega(D)}
$$

2.1. Congruences. The main goal in this subsection is to characterize congruences mod 2 and 4 for $H(4 n+3)$. The method of proof is completely analogous to the methods of [20], where the same type of results were found for certain weight $3 / 2$ eta-products. We provide analogues of the results in [20] needed to prove our characterizations of the mod 2 and mod 4 congruences for $H(4 n+3)$. These characterizations are given in Theorem 2.5.

We recall the following theorems.

Theorem 2.1. We have $3 H(3)=1$ and if $n$ is square-free, $n>3$ and $n \equiv 3(\bmod 4)$, then we have

$$
H(n)=h(-n)=2^{t-1} k,
$$

where $t$ is the number of distinct prime factors of $n$ and $k$ is the number of classes in each genus of $\mathbb{Q}(\sqrt{-n})$.

Remark. This theorem follows immediately from Proposition 3.11, Corollary 3.14 and Theorem 3.15 in [23].

Theorem 2.2. If $n$ is square-free and $n \equiv 3(\bmod 4)$, then we have

$$
\left(2-\left(\frac{n}{2}\right)\right) H(n)=\left(2-\left(\frac{n}{2}\right)\right) h(-n)=\sum_{r=1}^{(n-1) / 2}\left(\frac{r}{n}\right) .
$$

Remark. This theorem follows from [15, Theorem 3, p.346]. We note that

$$
\left(\frac{n}{2}\right)=\left\{\begin{array}{lll}
-1 & \text { if } n \equiv 3 \quad(\bmod 8) \\
1 & \text { if } n \equiv 7 & (\bmod 8)
\end{array}\right.
$$

The following is an analog of [20, Theorem 3.6].

Theorem 2.3. Suppose $n \equiv 3(\bmod 4)$ is square-free. We have 
(1) $3 H(n)$ is odd if and only if $n$ is a prime,

(2) $3 H(n) \equiv 2(\bmod 4)$ if and only if $n=p_{1} p_{2}$ is a product of two primes which satisfy

$$
\left(\frac{p_{1}}{p_{2}}\right)=-1
$$

Remark. The result (1) is well-known and follows from Theorem 2.1. The result (2) follows from Theorem 2.1 together with Propositions 3 and 4 in [37. It may also be proved using the method in [20, Section 3]. As pointed out by Pizer [37, p.189], (2) is also due to Hasse [27].

The Hurwitz class number function also satisfies the analog of [20, eq.(4.1)].

Lemma 2.4. For each odd prime $p$, we have

$$
H\left(p^{2} n\right)+\left(\frac{-n}{p}\right) H(n)+p H\left(n / p^{2}\right)=(p+1) H(n),
$$

for all $n \geq 0$.

Remark. It is understood that $H(n)=0$ if $n$ is non-integral or negative. This result is known. It is noted in the proof of Proposition 5.1 in [1. Ahlgren, Bringmann and Lovejoy [1] prove that the generating function for $H(n)$ is a Hecke eigenform by using the fact that the generating function is mock modular form of weight $3 / 2$. For completeness we provide an elementary proof that only uses (2.1). This argument was also observed by Beckwith, Raum and Richter [14].

Proof. Suppose $p$ is an odd prime. We may assume $n \equiv 0$ or $3(\bmod 4)$. Then, by (2.1) and (2.2), we have

$$
H(n)=H(-D) G(D, f)
$$

where $n=-D f^{2}, D$ is a fundamental discriminant of $\mathbb{Q}(\sqrt{-n})$, namely $-D$ is square-free or $-D=4 m, m \equiv 1,2(\bmod 4)$ and $m$ is square-free and

$$
G(D, f):=\sum_{d \mid f} \mu(d)\left(\frac{D}{d}\right) \sigma_{1}(f / d) .
$$

It is clear that $G(D, f)$ is a multiplicative function of $f$. We consider two cases.

Case 1. $(p, f)=1$. Then $n / p^{2} \notin \mathbb{Z}$ and $H\left(n / p^{2}\right)=0$.

$$
\begin{aligned}
H\left(p^{2} n\right) & =H(-D) G(D, p f)=H(-D) G(D, p) G(D, f) \\
& =H(-D)\left(\sigma_{1}(p)-\left(\frac{D}{p}\right)\right) G(D, f)=H(-D)\left(1+p-\left(\frac{D}{p}\right)\right) G(D, f) \\
& =-\left(\frac{D}{p}\right) H(n)+(1+p) H(n),
\end{aligned}
$$

so that (2.4) holds. 
Case 2. $(p, f)>1$. Then we let $f=p^{\alpha} f_{1}$ where $\alpha \geq 1$ and $\left(p, f_{1}\right)=1$. We have

$$
\begin{aligned}
& H\left(p^{2} n\right)=H(-D) G(D, p f)=H(-D) G\left(D, p^{\alpha+1}\right) G\left(D, f_{1}\right), \\
& H(n)=H(-D) G(D, f)=H(-D) G\left(D, p^{\alpha}\right) G\left(D, f_{1}\right), \\
& H\left(n / p^{2}\right)=H(-D) G(D, f / p)=H(-D) G\left(D, p^{\alpha-1}\right) G\left(D, f_{1}\right) .
\end{aligned}
$$

So we need to show

$$
G\left(D, p^{\alpha+1}\right)+p G\left(D, p^{\alpha-1}\right)-(1+p) G\left(D, p^{\alpha}\right)=0
$$

since $p \mid n$ and $\left(\frac{-n}{p}\right)=0$. This is an easy exercise since

$$
G\left(D, p^{\beta}\right)=\sigma_{1}\left(p^{\beta}\right)-\left(\frac{D}{p}\right) \sigma_{1}\left(p^{\beta}\right), \quad \text { and } \quad \sigma_{1}\left(p^{\beta+2}\right)=p^{\beta+2}+p^{\beta+1}+\sigma_{1}\left(p^{\beta}\right),
$$

for all $\beta>0$. We have $(2.4)$ in this case.

By Theorem 2.3 and Lemma 2.4, we have the following theorem by a proof that is analogous to that of Theorem 4.3 and Theorem 4.6 in [20].

Theorem 2.5. For $n \equiv 3(\bmod 4)$,

(1) $3 H(n)$ is odd if and only if $n$ has the form

$$
n=p^{4 a+1} m^{2},
$$

where $p$ is prime, and $m$ and $a$ are integers satisfying $(m, p)=1$ and $a \geq 0$.

(2) $3 H(n) \equiv 2(\bmod 4)$ if and only if $n$ has the form

$$
n=p_{1}^{4 a+1} p_{2}^{4 b+1} m^{2}
$$

where $p_{1}$ and $p_{2}$ are primes such that $\left(\frac{p_{1}}{p_{2}}\right)=-1,\left(m, p_{1} p_{2}\right)=1$ and $a, b \geq 0$ are integers.

Remark. The factor 3 that appears in Theorem 2.3 and Theorem 2.5 is only to guarantee that $3 H(n)$ is an integer.

2.2. Generating functions. In this subsection we give identities for certain generating functions

$$
\mathscr{H}_{a, b}(q):=\sum_{n=0}^{\infty} H(a n+b) q^{n}
$$

where $a$ is a divisor of 24 and $(a, b)=1$.

Gauss's Three Squares Theorem (see [34, Theorem 1.5]) shows that

$$
24 \mathscr{H}_{8,3}(q)=\sum_{n=0}^{\infty} r_{3}(8 n+3) q^{n}
$$


where $r_{3}(n)$ denotes the number of representations of $n$ as a sum of 3 squares. Noting that $x^{2}+y^{2}+z^{2} \equiv 3(\bmod 8)$ if and only if $x, y, z$ are odd, we have

$$
\begin{aligned}
\sum_{n=0}^{\infty} r_{3}(8 n+3) q^{8 n+3} & =\sum_{x, y, z \in \mathbb{Z}} q^{(2 x+1)^{2}+(2 y+1)^{2}+(2 z+1)^{2}}=8 \sum_{x, y, z \geq 0} q^{(2 x+1)^{2}+(2 y+1)^{2}+(2 z+1)^{2}} \\
& =8 q^{3} \sum_{x, y, z \geq 0} q^{4\left(x^{2}+x+y^{2}+y+z^{2}+z\right)}=8 q^{3}\left(\sum_{n=0}^{\infty} q^{4 n(n+1)}\right)^{3} .
\end{aligned}
$$

See also [40, Eq.(1.14), p.41]. Hence

$$
3 \mathscr{H}_{8,3}(q)=\left(\sum_{n=0}^{\infty} q^{n(n+1) / 2}\right)^{3}=\left(\frac{J_{2}^{2}}{J_{1}}\right)^{3}=\frac{J_{2}^{6}}{J_{1}^{3}}
$$

by (1.11). See also [40, Eq.(1.14), p.41]. By calculating the 3-dissection of $\mathscr{H}_{8,3}(q)$ and using (1.12) we find that

$$
\mathscr{H}_{24,11}(q)=\left(\sum_{n=-\infty}^{\infty} q^{n(3 n+1) / 2}\right)^{2} \sum_{n=0}^{\infty} q^{3 n(n+1) / 2}=\frac{J_{3}^{3} J_{2}^{2}}{J_{1}^{2}}
$$

and

$$
\mathscr{H}_{24,19}(q)=\sum_{n=-\infty}^{\infty} q^{n(3 n+1) / 2}\left(\sum_{n=0}^{\infty} q^{3 n(n+1) / 2}\right)^{2}=\frac{J_{6}^{3} J_{2}}{J_{1}} .
$$

We let $t(n)$ denote the number of representations of $n$ by the ternary quadratic form $x^{2}+$ $3 y^{2}+3 z^{2}$. Then Bringmann and Kane [17, p.3] found that

$$
t(n)=8\left(1+\left(\frac{n}{3}\right)\right) H(n),
$$

when $n \equiv 7(\bmod 8)$ with $9 \nmid n$. Equation (2.8) can also be proved using [17, Lemma 4.14] and Lemma 2.4. From equation (2.8) it can be shown that

$$
\mathscr{H}_{24,7}(q)=\sum_{n=-\infty}^{\infty} q^{n(3 n+1) / 2}\left(\sum_{n=0}^{\infty} q^{n(n+1) / 2}\right)^{2}=\frac{J_{3}^{2} J_{2}^{5}}{J_{6} J_{1}^{3}} .
$$

We omit the details. In [20] we obtained congruences modulo 4 for the coefficients of the eta-products in (2.6), (2.7) and (2.9) using special cases of Theorem 2.5.

We will need the following result of Humbert [30, p.368].

$$
\mathscr{H}_{8,7}(q)=\frac{1}{q J_{1}^{3}} \sum_{n=0}^{\infty} \frac{(-1)^{n+1} n^{2} q^{n(n+1) / 2}}{1+q^{n}} .
$$


See also [40, p.51]. Naturally we find that $\mathscr{H}_{8,7}(q)$ is not an eta-product. Humbert [30, p.442] also found

$$
\mathscr{H}_{24,23}(q)=q^{-23 / 24} \sum_{(x, y, z) \in D} q^{\left(2 x^{2}-3 z^{2}-6 y^{2}\right) / 24}
$$

where

$$
D=\left\{(x, y, z) \in \mathbb{Z}^{3}: 0<3 z<2 x,-x<3 y<x, 2 x^{2}-3 z^{2}-6 y^{2} \equiv 23 \quad(\bmod 24)\right\} .
$$

\section{Congruences for A ClAss OF MOCK THETA FunCtions}

In this section we will discuss congruences for four mock theta functions associated with the Hurwitz class number functions $\mathscr{H}_{4,3}, \mathscr{H}_{8,7}, \mathscr{H}_{12,11}$ and $\mathscr{H}_{24,23}$. These mock theta functions are $A(q)$ (second order), $\phi_{-}(q)$ and $\sigma(q)$ (sixth order), and $V_{1}(q)$ (eighth order). The parity of the coefficients of these functions and many other mock theta functions was recently found by Wang [42]. We determine their behaviour mod 4 by relating them with the Hurwitz class number.

3.1. The second order mock theta function $A(q)$. The second order mock theta function $A(q)$ is defined in (1.7). We find a congruence relation between the coefficients of $A(q)$ and the Hurwitz class number $H(8 n-1)$. We have the following lemma.

Lemma 3.1. For each integer $n>0$, we have

$$
N_{A}(n) \equiv(-1)^{n+1} H(8 n-1) \quad(\bmod 4) .
$$

Proof. Ramanujan [38, p.8] found that $A(q)$ can be written as an Appell-Lerch sum

$$
A(q)=q \frac{\left(-q ; q^{2}\right)_{\infty}}{\left(q^{2} ; q^{2}\right)_{\infty}} \sum_{n=0}^{\infty} \frac{(-1)^{n} q^{2 n^{2}+3 n}}{1-q^{2 n+1}} .
$$

For a proof see [6, p.265]. Combining $(2.10)$ and $(3.2)$ and noting that $(2 n)^{2} \equiv 0(\bmod 4)$ and $(2 n+1)^{2} \equiv 1(\bmod 4)$, we have

$$
\begin{aligned}
\mathscr{H}_{8,7}(q) & =\frac{1}{q J_{1}^{3}} \sum_{n=0}^{\infty} \frac{(-1)^{n+1} n^{2} q^{n(n+1) / 2}}{1+q^{n}} \equiv \frac{J_{1}^{4}}{J_{2}^{2}} \cdot \frac{1}{q J_{1}^{3}} \sum_{n=0}^{\infty} \frac{(2 n+1)^{2} q^{(2 n+1)(n+1)}}{1+q^{2 n+1}} \\
& \equiv \frac{J_{1}}{J_{2}^{2}} \sum_{n=0}^{\infty} \frac{q^{2 n^{2}+3 n}}{1+q^{2 n+1}}=\frac{A(-q)}{-q} \quad(\bmod 4),
\end{aligned}
$$

by 1.9 . This implies

$$
(-1)^{n+1} N_{A}(n) \equiv H(8 n-1) \quad(\bmod 4),
$$

which is 3.1 .

We note that by (3.1), $N_{A}(n)$ is odd if and only if $H(8 n-1)$ is odd and $N_{A}(n) \equiv 2$ $(\bmod 4)$ if and only if $H(8 n-1) \equiv 2(\bmod 4)$. Also $m^{2} \equiv 1(\bmod 8)$ for each odd $m$. Thus Theorem 2.5 implies 
Theorem 3.2. Let $n$ be a positive integer.

(1) $N_{A}(n)$ is odd if and only if $8 n-1$ has the form

$$
8 n-1=p^{4 a+1} m^{2},
$$

where $p$ is prime, and $m$ and $a$ are integers satisfying $(m, p)=1$ and $a \geq 0$.

(2) $N_{A}(n) \equiv 2(\bmod 4)$ if and only if $8 n-1$ has the form

$$
8 n-1=p_{1}^{4 a+1} p_{2}^{4 b+1} m^{2},
$$

where $p_{1}$ and $p_{2}$ are primes such that $\left(\frac{p_{1}}{p_{2}}\right)=-1,\left(m, p_{1} p_{2}\right)=1$ and $a, b \geq 0$ are integers.

Remark. Wang [42, Theorem 3.1] proved (1) by a different method.

Theorem 3.3. Kim, Lim and Lovejoy's Conjecture 1.4 is true.

Proof. Let $p \not \equiv 7(\bmod 8)$ be an odd prime and suppose $n, k$ are nonnegative integers where $\left(\frac{k}{p}\right)=1$. Suppose $m=p^{2} n+(p k+1) \delta_{p}$, where $\delta_{p}=\frac{1}{8}\left(7 p^{2}+1\right)$. Since

$$
8 m-1 \equiv p k \quad\left(\bmod p^{2}\right),
$$

we have $p \| 8 m-1$. Theorem 3.2(1) implies that $N_{A}(m)$ is even since $p \not \equiv 7(\bmod 8)$. Now suppose $N_{A}(m) \equiv 2(\bmod 4)$. Then Theorem $3.2(2)$ and 3.4 imply that

$$
8 m-1=p^{1} q^{4 b+1} t^{2}, \quad \text { and } \quad k \equiv q^{4 b+1} t^{2} \quad(\bmod p),
$$

where $q$ is a prime satisfying $\left(\frac{p}{q}\right)=-1,(p q, t)=1$, and $b \geq 0, t>0$ are integers. Since $p q \equiv-1(\bmod 8)$ either $p$ or $q \equiv 1(\bmod 4)$ so by quadratic reciprocity $\left(\frac{q}{p}\right)=\left(\frac{p}{q}\right)=-1$. But

$$
\left(\frac{q}{p}\right)=\left(\frac{q^{4 b+1} t^{2}}{p}\right)=\left(\frac{k}{p}\right)=1
$$

which is a contradiction. Hence $N_{A}(m) \not \equiv 2(\bmod 4)$, and

$$
N_{A}(m) \equiv 0 \quad(\bmod 4) \text {. }
$$

3.2. The eighth order mock theta function $V_{1}(q)$. McIntosh [33] studied the eighth order mock theta function

$$
V_{1}(q)=\sum_{n=0}^{\infty} N_{V_{1}}(n) q^{n}=\sum_{n=0}^{\infty} \frac{q^{(n+1)^{2}}\left(-q ; q^{2}\right)_{n}}{\left(q ; q^{2}\right)_{n+1}} .
$$

By [33, p.286 Eq(4),Eq(7)] we have

$$
V_{1}(q)=A\left(q^{2}\right)+q P\left(q^{2}\right),
$$


where

$$
P(q)=(-q ; q)_{\infty}\left(-q^{2} ; q^{2}\right)_{\infty}^{2}\left(q^{4} ; q^{4}\right)_{\infty}=\frac{J_{4}^{3}}{J_{1} J_{2}}
$$

We have the following lemma for $N_{V_{1}}(n)$.

Lemma 3.4. For each integer $n>0$, we have

$$
N_{V_{1}}(n) \equiv \chi(n) H(4 n-1) \quad(\bmod 4),
$$

where $\chi(n)=-1$ if $n \equiv 0,1(\bmod 4)$ and $\chi(n)=1$ if $n \equiv 2,3(\bmod 4)$.

Proof. Equations (3.1) and (3.5) give

$$
N_{V_{1}}(2 n)=N_{A}(n) \equiv(-1)^{n+1} H(8 n-1) \quad(\bmod 4),
$$

which implies that (3.7) holds for even $n$. By 1.9$),(2.5)$ and $(3.6)$ we have

$$
P(-q)=(q ;-q)_{\infty}\left(-q^{2} ; q^{2}\right)_{\infty}^{2}\left(q^{4} ; q^{4}\right)_{\infty}=\frac{J_{4}^{4} J_{1}^{4}}{J_{2}^{10}} \cdot \frac{J_{2}^{6}}{J_{1}^{3}} \equiv \frac{J_{2}^{6}}{J_{1}^{3}}=3 \mathscr{H}_{8,3}(q) \quad(\bmod 4) .
$$

Hence

$$
N_{V_{1}}(2 n+1) \equiv(-1)^{n+1} H(8 n+3) \quad(\bmod 4),
$$

which implies that (3.7) holds for odd $n$.

By (3.7), $N_{V_{1}}(n)$ is odd if and only if $H(4 n-1)$ is odd and $N_{V_{1}}(n) \equiv 2(\bmod 4)$ if and only if $H(4 n-1) \equiv 2(\bmod 4)$. From Theorem 2.5 we obtain

Theorem 3.5. For any positive integer $n$,

(1) $N_{V_{1}}(n)$ is odd if and only if $4 n-1$ has the form

$$
4 n-1=p^{4 a+1} m^{2}
$$

where $p$ is prime, and $m$ and $a$ are integers satisfying $(m, p)=1$ and $a \geq 0$.

(2) $N_{V_{1}}(n) \equiv 2(\bmod 4)$ if and only if $4 n-1$ has the form

$$
4 n-1=p_{1}^{4 a+1} p_{2}^{4 b+1} m^{2},
$$

where $p_{1}$ and $p_{2}$ are primes such that $\left(\frac{p_{1}}{p_{2}}\right)=-1,\left(m, p_{1} p_{2}\right)=1$ and $a, b \geq 0$ are integers.

Remark. The result (1) was also found by Wang [42, Theorem 8.4].

Theorem 3.6. Let $p \not \equiv 3(\bmod 4)$ be an odd prime, suppose $4 \delta_{p} \equiv 1\left(\bmod p^{2}\right)$ and $k, n \in \mathbb{Z}$ where $\left(\frac{k}{p}\right)=1$. Then

$$
N_{V_{1}}\left(p^{2} n+(p k+1) \delta_{p}\right) \equiv 0 \quad(\bmod 4) .
$$


Proof. Let $p \not \equiv 3(\bmod 4)$ be an odd prime and suppose $n, k$ are nonnegative integers where $\left(\frac{k}{p}\right)=1$. Suppose $m=p^{2} n+(p k+1) \delta_{p}$, where $\delta_{p}=\frac{1}{4}\left(3 p^{2}+1\right)$. Since

$$
4 m-1 \equiv p k \quad\left(\bmod p^{2}\right),
$$

we have $p \| 4 m-1$. Theorem $3.5(1)$ implies that $N_{V_{1}}(m)$ is even since $p \not \equiv 3(\bmod 4)$. Now suppose $N_{V_{1}}(m) \equiv 2(\bmod 4)$. Then Theorem 3.5 $(2)$ and $(3.8)$ imply that

$$
4 m-1=p^{1} q^{4 b+1} t^{2}, \quad \text { and } \quad k \equiv q^{4 b+1} t^{2} \quad(\bmod p)
$$

where $q$ is a prime satisfying $\left(\frac{p}{q}\right)=-1,(p q, t)=1$, and $b \geq 0, t>0$ are integers. Since $p q \equiv-1(\bmod 4)$ either $p$ or $q \equiv 1(\bmod 4)$ so by quadratic reciprocity $\left(\frac{q}{p}\right)=\left(\frac{p}{q}\right)=-1$. But

$$
\left(\frac{q}{p}\right)=\left(\frac{q^{4 b+1} t^{2}}{p}\right)=\left(\frac{k}{p}\right)=1
$$

which is a contradiction. Hence $N_{V_{1}}(m) \not \equiv 2(\bmod 4)$, and

$$
N_{V_{1}}(m) \equiv 0 \quad(\bmod 4)
$$

3.3. The sixth order mock theta function $\phi_{-}(q)$. Berndt and Chan [13] defined two new sixth order mock theta functions including

$$
\phi_{-}(q)=\sum_{n=1}^{\infty} N_{\phi_{-}}(n) q^{n}:=\sum_{n=1}^{\infty} \frac{q^{n}(-q ; q)_{2 n-1}}{\left(q, q^{2}\right)_{n}} .
$$

We will show that $\phi_{-}(q)$ is associated with $\mathscr{H}_{24,23}$. This is more difficult than showing $A(q)$ is associated with $\mathscr{H}_{8,7}$. Unfortunately Humbert's formula (2.11) did not reveal the relations needed. Instead we find a relation mod 4 between $\phi_{-}(q)$ and part of the 3 -dissection of $A(-q)$. Following Hickerson and Mortenson [29] we define

$$
j(z ; q):=(z ; q)_{\infty}\left(z^{-1} q ; q\right)_{\infty}(q ; q)_{\infty},
$$

and

$$
m(x, q, z):=\frac{1}{j(z ; q)} \sum_{r=-\infty}^{\infty} \frac{(-1)^{r} q^{r(r-1) / 2} z^{r}}{1-q^{r-1} x z} .
$$

Hickerson and Mortenson used $m(x, q, z)$ as a building block for expressing the mock theta functions in terms of Appell-Lerch sums. They found

$$
A(q)=-m\left(q, q^{4}, q^{2}\right), \quad([29, \text { Eq.(5.1),p.399]) }
$$

and

$$
\phi_{-}(q)=-m\left(q, q^{3}, q\right)-q \frac{J_{6}^{6}}{J_{2}^{2} J_{3}^{3}}, \quad([29, \text { Eq.(5.30),p.401]. })
$$


We define the usual Atkin $U_{p}$ operator which acts on a formal power series

$$
f(q)=\sum_{n \in \mathbb{Z}} a(n) q^{n}
$$

by

$$
U_{p}(f(q))=\sum_{n \in \mathbb{Z}} a(p n) q^{n}
$$

Lemma 3.7. We have

$$
U_{3}(A(-q))=\phi_{-}(q)-4 q \frac{J_{6}^{2} J_{4}^{8} J_{1}^{2}}{J_{3} J_{2}^{10}}
$$

so that

$$
U_{3}(A(-q)) \equiv \phi_{-}(q) \quad(\bmod 4)
$$

Proof. Replacing $q$ by $q^{4}$ and setting $x=-q$ in [29, Corollary 3.8], we have

$$
\begin{aligned}
m\left(-q, q^{4},-1\right)= & m\left(-q^{15}, q^{36},-1\right)+\frac{1}{q^{3}} m\left(-q^{3}, q^{36},-1\right) \\
& +\frac{1}{q^{10}} m\left(-q^{-9}, q^{36},-1\right)-\frac{1}{2 q^{3}} \frac{J_{36} J_{24} J_{12} J_{6} J_{2}}{J_{72}^{2} J_{8} J_{3}},
\end{aligned}
$$

after simplification. Replacing $q$ by $q^{4}$ and setting $x=-q, z_{0}=q^{2}$ and $z_{1}=-1$ in [29, Theorem 3.3], we have

$$
m\left(-q, q^{4},-1\right)=m\left(-q, q^{4}, q^{2}\right)+\frac{J_{4}^{10} J_{1}}{2 J_{8}^{4} J_{2}^{6}},
$$

after simplification. Hence by (3.9), (3.12) and (3.13)

$$
U_{3}(-A(-q))=m\left(-q^{5}, q^{12},-1\right)+\frac{1}{q} m\left(-q, q^{12},-1\right)-U_{3}(G(q)),
$$

where

$$
G(q)=\frac{1}{2}\left(\frac{J_{36} J_{24} J_{12} J_{6} J_{2}}{q^{3} J_{72}^{2} J_{8} J_{3}}+\frac{J_{4}^{10} J_{1}}{J_{8}^{4} J_{2}^{6}}\right) .
$$

The following identities may be proved using the theory of modular functions by verifying that both sides hold for a sufficient number of terms:

$$
U_{3}\left(\frac{J_{24}^{3} J_{2}}{J_{36} J_{18} J_{12} J_{8}}\right)=1, \quad U_{3}\left(\frac{J_{24}^{4} J_{4}^{10} J_{9} J_{6}^{12} J_{1}}{J_{18}^{2} J_{12}^{10} J_{8}^{4} J_{3}^{6} J_{2}^{6}}\right)=1 .
$$

This verification was carried out using the second author's maple ETA package, which is available at

$$
\text { https://qseries.org/fgarvan/qmaple/ETA/ }
$$


The two functions involved can be rewritten in terms of eta-products, which turn out to be modular functions on $\Gamma_{0}(72)$. We then deduce that

$$
U_{3}\left(\frac{J_{36} J_{24} J_{12} J_{6} J_{2}}{q^{3} J_{72}^{2} J_{8} J_{3}}\right)=\frac{J_{12}^{2} J_{6} J_{4}^{2} J_{2}}{q J_{24}^{2} J_{8}^{2} J_{1}}, \quad U_{3}\left(\frac{J_{4}^{10} J_{1}}{J_{8}^{4} J_{2}^{6}}\right)=\frac{J_{6}^{2} J_{4}^{10} J_{1}^{6}}{J_{8}^{4} J_{3} J_{2}^{12}} .
$$

Substituting 3.15 into 3.14, we have

$$
U_{3}(-A(-q))=m\left(-q^{5}, q^{12},-1\right)+\frac{1}{q} m\left(-q, q^{12},-1\right)-\frac{J_{12}^{2} J_{6} J_{4}^{2} J_{2}}{2 q J_{24}^{2} J_{8}^{2} J_{1}}-\frac{J_{6}^{2} J_{4}^{10} J_{1}^{6}}{2 J_{8}^{4} J_{3} J_{2}^{12}},
$$

Replacing $q$ by $q^{3}$ and setting $x=z=q^{2}$ in [29, Corollary 3.7], we have

$$
m\left(q^{2}, q^{3}, q^{2}\right)=m\left(-q^{7}, q^{12}, q^{8}\right)-\frac{1}{q} m\left(-q, q^{12}, q^{8}\right)+2 q \frac{J_{12}^{3} J_{3}^{3} J_{2}}{J_{6}^{3} J_{4} J_{1}^{2}},
$$

after simplification. Since the equations in [29, Proposition 3.1] imply that

$$
m(x, q, z)+m(q / x, q, q / z)=1,
$$

we can rewrite $(3.17)$ as

$$
m\left(q, q^{3}, q\right)=m\left(-q^{5}, q^{12}, q^{4}\right)+\frac{1}{q} m\left(-q, q^{12}, q^{8}\right)-2 q \frac{J_{12}^{3} J_{3}^{3} J_{2}}{J_{6}^{3} J_{4} J_{1}^{2}} .
$$

Again using [29, Theorem 3.3] we have

$$
\begin{gathered}
m\left(-q^{5}, q^{12},-1\right)=m\left(-q^{5}, q^{12}, q^{4}\right)+P_{1}(q), \\
m\left(-q, q^{12},-1\right)=m\left(-q, q^{12}, q^{8}\right)+P_{2}(q),
\end{gathered}
$$

where

$$
\begin{aligned}
& P_{1}(q)=\frac{J_{12}^{3} j\left(-q^{4} ; q^{12}\right) j\left(q^{9} ; q^{12}\right)}{j\left(q^{4} ; q^{12}\right) j\left(-1 ; q^{12}\right) j\left(-q^{9} ; q^{12}\right) j\left(q^{5} ; q^{12}\right)}, \\
& P_{2}(q)=\frac{J_{12}^{3} j\left(-q^{4} ; q^{12}\right) j\left(q^{9} ; q^{12}\right)}{j\left(q^{4} ; q^{12}\right) j\left(-1 ; q^{12}\right) j\left(-q^{9} ; q^{12}\right) j\left(q ; q^{12}\right)} .
\end{aligned}
$$

We have

$$
P_{1}(q)+\frac{1}{q} P_{2}(q)=\frac{J_{12}^{3} j\left(-q^{4}, q^{12}\right) j\left(q^{9}, q^{12}\right)}{q j\left(q^{4}, q^{12}\right) j\left(-1, q^{12}\right) j\left(-q^{9}, q^{12}\right) j\left(q^{5}, q^{12}\right) j\left(q, q^{12}\right)}\left(j\left(q^{5}, q^{12}\right)+q j\left(q, q^{12}\right) .\right.
$$

In the quintuple product identity 1.13 quintprod we let $z=q$ and replace $q$ by $q^{4}$ to find that

$$
j\left(q^{5}, q^{12}\right)+q j\left(q, q^{12}\right)=\left(-q ; q^{2}\right)_{\infty}\left(q^{2} ; q^{2}\right)_{\infty} .
$$

Equations 3.21, P12ida and 3.22 quinpapp give the following identity

$$
P_{1}(q)+\frac{1}{q} P_{2}(q)=\frac{J_{12}^{5} J_{8} J_{3}^{3} J_{2}^{4}}{2 q J_{24}^{3} J_{6}^{4} J_{4}^{3} J_{1}^{2}}
$$


after some simplification using Jacobi's triple product identity (1.10). Combining (3.10), (3.16) and (3.18)-(3.23), we have

$$
U_{3}(A(-q))=\phi_{-}(q)+\frac{J_{12}^{2} J_{6} J_{4}^{2} J_{2}}{2 q J_{24}^{2} J_{8}^{2} J_{1}}+\frac{J_{6}^{2} J_{4}^{10} J_{1}^{6}}{2 J_{8}^{4} J_{3} J_{2}^{12}}+\frac{q J_{6}^{6}}{J_{3}^{3} J_{2}^{2}}-\frac{J_{12}^{5} J_{8} J_{3}^{3} J_{2}^{4}}{2 q J_{24}^{3} J_{6}^{4} J_{4}^{3} J_{1}^{2}}-\frac{2 q J_{12}^{3} J_{3}^{3} J_{2}}{J_{6}^{3} J_{4} J_{1}^{2}}
$$

Finally we have

$$
\frac{J_{12}^{2} J_{6} J_{4}^{2} J_{2}}{2 q J_{24}^{2} J_{8}^{2} J_{1}}+\frac{J_{6}^{2} J_{4}^{10} J_{1}^{6}}{2 J_{8}^{4} J_{3} J_{2}^{12}}+q \frac{J_{6}^{6}}{J_{3}^{3} J_{2}^{2}}-\frac{J_{12}^{5} J_{8} J_{3}^{3} J_{2}^{4}}{2 q J_{24}^{3} J_{6}^{4} J_{4}^{3} J_{1}^{2}}-2 q \frac{J_{12}^{3} J_{3}^{3} J_{2}}{J_{6}^{3} J_{4} J_{1}^{2}}=-4 q \frac{J_{6}^{2} J_{4}^{8} J_{1}^{2}}{J_{3} J_{2}^{10}} .
$$

This can be proved using the maple ETA package. The identity can be rewritten as an identity for eta-products that are modular functions on $\Gamma_{0}(24)$. Equation (3.11) follows from (3.24) and (3.25). The mod 4 congruence follows immediately.

Lemmas 3.1 and 3.7 imply the following

Lemma 3.8. Let $n$ be a positive integer.

$$
N_{\phi_{-}}(n) \equiv-H(24 n-1) \quad(\bmod 4),
$$

We note that if $(m, 6)=1$ then $m^{2} \equiv 1(\bmod 24)$. Thus the following theorem follows easily from Lemma 3.8 and Theorem 2.5 .

Theorem 3.9. For $n>0$ be an integer,

(1) $N_{\phi_{-}}(n)$ is odd if and only if $24 n-1$ has the form

$$
24 n-1=p^{4 a+1} m^{2},
$$

where $p$ is prime, and $m$ and $a$ are integers satisfying $(m, p)=1$ and $a \geq 0$.

(2) $N_{\phi_{-}}(n) \equiv 2(\bmod 4)$ if and only if $24 n-1$ has the form

$$
24 n-1=p_{1}^{4 a+1} p_{2}^{4 b+1} m^{2},
$$

where $p_{1}$ and $p_{2}$ are primes such that $\left(\frac{p_{1}}{p_{2}}\right)=-1,\left(m, p_{1} p_{2}\right)=1$ and $a, b \geq 0$ are integers.

Remark. Wang [42, Theorem 6.6] proved (1) by showing that

$$
N_{\phi_{-}}(n) \equiv N_{\psi}(n) \quad(\bmod 2) .
$$

Theorem 3.10. Let $p>3$ be a prime and $p \not \equiv 23(\bmod 24)$. Suppose $24 \delta_{p} \equiv 1\left(\bmod p^{2}\right)$ and $k, n \in \mathbb{Z}$ where $\left(\frac{k}{p}\right)=1$. Then

$$
N_{\phi_{-}}\left(p^{2} n+(p k+1) \delta_{p}\right) \equiv 0 \quad(\bmod 4)
$$


Proof. Let $p \not \equiv 23(\bmod 24)$ be a prime $>3$, and suppose $n, k$ are nonnegative integers where $\left(\frac{k}{p}\right)=1$. Suppose $m=p^{2} n+(p k+1) \delta_{p}$, where $\delta_{p}=\frac{1}{24}\left(23 p^{2}+1\right)$. Since

$$
24 m-1 \equiv p k \quad\left(\bmod p^{2}\right),
$$

we have $p \| 24 m-1$. Theorem $3.9(1)$ implies that $N_{\phi_{-}}(m)$ is even since $p \not \equiv 23(\bmod 24)$. Now suppose $N_{\phi_{-}}(m) \equiv 2(\bmod 4)$. Then Theorem 3.9 $(2)$ and $(3.27)$ imply that

$$
24 m-1=p^{1} q^{4 b+1} t^{2}, \quad \text { and } \quad k \equiv q^{4 b+1} t^{2} \quad(\bmod p),
$$

where $q$ is a prime satisfying $\left(\frac{p}{q}\right)=-1,(p q, t)=1$, and $b \geq 0, t>0$ are integers. Since $p q \equiv-1(\bmod 24)$ either $p$ or $q \equiv 1(\bmod 4)$ so by quadratic reciprocity $\left(\frac{q}{p}\right)=\left(\frac{p}{q}\right)=-1$. But

$$
\left(\frac{q}{p}\right)=\left(\frac{q^{4 b+1} t^{2}}{p}\right)=\left(\frac{k}{p}\right)=1
$$

which is a contradiction. Hence $N_{\phi_{-}}(m) \not \equiv 2(\bmod 4)$, and

$$
N_{\phi_{-}}(m) \equiv 0 \quad(\bmod 4) \text {. }
$$

3.4. The sixth order mock theta function $\sigma(q)$. Ramanujan's sixth order mock theta function

$$
\sigma(q):=\sum_{n=0}^{\infty} N_{\sigma}(n) q^{n}:=\sum_{n=0}^{\infty} \frac{q^{(n+1)(n+2) / 2}(-q ; q)_{n}}{\left(q ; q^{2}\right)_{n+1}}
$$

was first studied by Andrews and Hickerson [9]. By [13, Theorem 1.1] we have

$$
\sigma(q)=\phi_{-}\left(q^{2}\right)+q P_{3}\left(q^{2}\right),
$$

where

$$
P_{3}(q)=(-q ; q)_{\infty}^{2}\left(-q^{3},-q^{3}, q^{3} ; q^{3}\right)_{\infty}=\frac{J_{6}^{2} J_{2}^{2}}{J_{3} J_{1}^{2}}
$$

The following lemma follows from Lemma 3.8 together with equations 3.28 and 2.6 .

Lemma 3.11. For each integer $n>0$, we have

$$
N_{\sigma}(n) \equiv(-1)^{n+1} H(12 n-1) \quad(\bmod 4) .
$$

Proof. By Lemma 3.8 and equation (3.28) we have

$$
N_{\sigma}(2 n)=N_{\phi_{-}}(n) \equiv-H(24 n-1) \quad(\bmod 4),
$$

which implies (3.30 for even $n$.

By 1.9), 2.6) and 3.29) we have

$$
P_{3}(q)=\frac{J_{6}^{2} J_{2}^{2}}{J_{3} J_{1}^{2}}=\frac{J_{3}^{3} J_{2}^{2}}{J_{1}^{2}} \cdot\left(\frac{J_{6}}{J_{3}^{2}}\right)^{2} \equiv \frac{J_{3}^{3} J_{2}^{2}}{J_{1}^{2}}=\mathscr{H}_{24,11}(q) \quad(\bmod 4) .
$$


Hence

$$
N_{\sigma}(2 n+1) \equiv H(24 n+11) \quad(\bmod 4)
$$

and (3.30) holds for odd $n$.

Again we note that if $(m, 6)=1$ then $m^{2} \equiv 1(\bmod 12)$. Thus the following theorem follows easily from Lemma 3.11 and Theorem 2.5.

Theorem 3.12. For $n>0$ be an integer,

(1) $N_{\sigma}(n)$ is odd if and only if $12 n-1$ has the form

$$
12 n-1=p^{4 a+1} m^{2},
$$

where $p$ is prime, and $m$ and $a$ are integers satisfying $(m, p)=1$ and $a \geq 0$.

(2) $N_{\sigma}(n) \equiv 2(\bmod 4)$ if and only if $12 n-1$ has the form

$$
12 n-1=p_{1}^{4 a+1} p_{2}^{4 b+1} m^{2},
$$

where $p_{1}$ and $p_{2}$ are primes such that $\left(\frac{p_{1}}{p_{2}}\right)=-1,\left(m, p_{1} p_{2}\right)=1$ and $a, b \geq 0$ are integers.

Remark. Wang [42, Theorem 6.4] proved (1) by using a Hecke-Rogers series identity of Andrews and Hickerson [9].

Theorem 3.13. Let $p>3$ be a prime and $p \not \equiv 11(\bmod 12)$. Suppose $12 \delta_{p} \equiv 1\left(\bmod p^{2}\right)$ and $k, n \in \mathbb{Z}$ where $\left(\frac{k}{p}\right)=1$. Then

$$
N_{\sigma}\left(p^{2} n+(p k+1) \delta_{p}\right) \equiv 0 \quad(\bmod 4) .
$$

Proof. Let $p \not \equiv 11(\bmod 12)$ be a prime $>3$, and suppose $n, k$ are nonnegative integers where $\left(\frac{k}{p}\right)=1$. Suppose $m=p^{2} n+(p k+1) \delta_{p}$, where $\delta_{p}=\frac{1}{12}\left(11 p^{2}+1\right)$. Since

$$
12 m-1 \equiv p k \quad\left(\bmod p^{2}\right),
$$

we have $p \| 12 m-1$. Theorem $3.12(1)$ implies that $N_{\sigma}(m)$ is even since $p \not \equiv 23(\bmod 24)$. Now suppose $N_{\sigma}(m) \equiv 2(\bmod 4)$. Then Theorem 3.12(2) and (3.31) imply that

$$
12 m-1=p^{1} q^{4 b+1} t^{2}, \quad \text { and } \quad k \equiv q^{4 b+1} t^{2} \quad(\bmod p),
$$

where $q$ is a prime satisfying $\left(\frac{p}{q}\right)=-1,(p q, t)=1$, and $b \geq 0, t>0$ are integers. Since $p q \equiv-1(\bmod 12)$ either $p$ or $q \equiv 1(\bmod 4)$ so by quadratic reciprocity $\left(\frac{q}{p}\right)=\left(\frac{p}{q}\right)=-1$. However

$$
\left(\frac{q}{p}\right)=\left(\frac{q^{4 b+1} t^{2}}{p}\right)=\left(\frac{k}{p}\right)=1,
$$

which is a contradiction. Hence $N_{\sigma}(m) \not \equiv 2(\bmod 4)$, and

$$
N_{\sigma}(m) \equiv 0 \quad(\bmod 4) \text {. }
$$




\section{Mod 4 COnjeCtures For unimodal SEQuences And THE Andrews SPT-FUNCTION}

In Section 3 we derived mod 4 congruences for four mock theta functions that are closely associated with the Hurwitz class number. In this section we prove the corrected version of Kim, Lim and Lovejoy's mod 4 conjectures for odd-balanced unimodal sequences, the second author's mod 4 conjectures for the Andrews's spt-function and Bryson, Ono, Pitman, and Rhoades's mod 4 conjectures for strongly unimodal sequences. The proofs depend on some of the mod 4 congruences for certain mock theta functions in the previous section, as well as some new Hecke-Rogers identities.

4.1. Number of partitions in odd-balanced unimodal sequences. Recall from Section 1 that

$$
\mathcal{V}(z ; q):=\sum_{m, n} v(m, n) z^{m} q^{n}=\sum_{n=0}^{\infty} \frac{(-z q ; q)_{n}\left(-z^{-1} q ; q\right)_{n} q^{n}}{\left(q ; q^{2}\right)_{n+1}} .
$$

is the two-parameter generating function for odd-balanced unimodal sequences of size $2 n+2$ and rank $m$.

By [36, p.258] we have

$$
\begin{aligned}
\left(1+\frac{1}{z}\right) \mathcal{V}(z, q) & =\frac{(-q ; q)_{\infty}}{(q ; q)_{\infty}}\left(\sum_{n, r \geq 0}-\sum_{n, r<0}\right)(-1)^{n} z^{r} q^{n^{2}+2 n+(2 n+1) r+r(r+1) / 2} \\
& =\frac{(-q ; q)_{\infty}}{(q ; q)_{\infty}} \sum_{n, r \geq 0}(-1)^{n}\left(z^{r}+z^{-r-1}\right) q^{n^{2}+2 n+(2 n+1) r+r(r+1) / 2} \\
& =\frac{(-q ; q)_{\infty}}{(q ; q)_{\infty}} \sum_{n, r \geq 0}(-1)^{n}\left(z^{r}+z^{-r-1}\right) q^{(n+r)^{2}+2(n+r)-r(r+1) / 2} \\
& =\frac{(-q ; q)_{\infty}}{(q ; q)_{\infty}} \sum_{n}^{\infty} \sum_{r=0}^{n}(-1)^{n+r}\left(z^{r}+z^{-r-1}\right) q^{n^{2}+2 n-r(r+1) / 2} .
\end{aligned}
$$

See also [31, Eq (1.12)]. Hence letting $z=1$ we find that

$$
\sum_{n=0}^{\infty} v(n) q^{n}=\mathcal{V}(1, q)=\frac{(-q ; q)_{\infty}}{(q ; q)_{\infty}} \sum_{n=0}^{\infty} \sum_{r=0}^{n}(-1)^{n+r} q^{n^{2}+2 n-r(r+1) / 2}
$$

where $v(n)$ is the number of odd-balanced unimodal sequences of size $2 n+2$. Letting $z=i$ in (4.1) and (4.2) we have

$$
\frac{A(q)}{q}=\mathcal{V}(i, q)=\frac{(-q ; q)_{\infty}}{(q ; q)_{\infty}} \sum_{n=0}^{\infty} \sum_{r=0}^{n}(-1)^{n+r+r(r+1) / 2} q^{n^{2}+2 n-r(r+1) / 2}
$$


by (1.7), after some simplification. The form of the series in equations (4.3) and (4.4) is quite similar. It is easy see that

$$
v(n) \equiv N_{A}(n+1) \quad(\bmod 2) .
$$

As expected Theorem 3.2(1) confirms the equivalent [31, Theorem 1.1]. We now consider $v(n) \bmod 4$. In this section we prove

Theorem 4.1. Let $p \not \equiv \pm 1(\bmod 8)$ be an odd prime, suppose $8 \delta_{p} \equiv 1\left(\bmod p^{2}\right)$ and $k, n \in \mathbb{Z}$ where $\left(\frac{k}{p}\right)=1$. Then

$$
v\left(p^{2} n+(p k-7) \delta_{p}\right) \equiv 0 \quad(\bmod 4) .
$$

Remark. We have corrected Kim, Lim and Lovejoy's original Conjecture 1.2 .

We define

$$
D_{v}(q):=\sum_{n=0}^{\infty} d_{v}(n) q^{n}:=\sum_{m=0}^{\infty} \sum_{r=0}^{m} q^{m^{2}+2 m-r(r+1) / 2} .
$$

Lemma 4.2. If $p \equiv 3,5(\bmod 8)$ is prime and $p \| 8 n+7$ then $d_{v}(n)=0$.

Proof. Suppose that $p \equiv 3,5(\bmod 8)$ is prime and $p \| 8 n+7$. Suppose by way of contradiction that $d_{v}(n) \neq 0$. Then

$$
8 n+7=8\left(m^{2}+2 m-r(r+1) / 2\right)+7=8(m+1)^{2}-(2 r+1)^{2},
$$

for some integers $m \geq 0$ and $0 \leq r \leq m$. Since $p \mid 8 n+7$ this implies

$$
8(m+1)^{2} \equiv(2 r+1)^{2} \quad(\bmod p) .
$$

Since $p \equiv 3,5(\bmod 8),\left(\frac{8}{p}\right)=-1$ and $(m+1) \equiv(2 r+1) \equiv 0(\bmod p)$. But this implies $p^{2} \mid 8 n+7$, which contradicts $p \| 8 n+7$. We conclude that $d_{v}(n)=0$.

Proof of Theorem 4.1. Let

$$
D_{0}(q):=\mathcal{V}(1, q)-\frac{A(q)}{q}=\sum_{n=0}^{\infty}\left(v(n)-N_{A}(n+1)\right) q^{n}
$$

By (4.3) and (4.4) we have

$$
\begin{aligned}
D_{0}(q) & =\frac{(-q ; q)_{\infty}}{(q ; q)_{\infty}} \sum_{n=0}^{\infty} \sum_{r=0}^{n}(-1)^{n+r}\left(1-(-1)^{r(r+1) / 2}\right) q^{n^{2}+2 n-r(r+1) / 2} \\
& =2 \frac{(-q ; q)_{\infty}}{(q ; q)_{\infty}} \sum_{n=0}^{\infty} \sum_{r=0}^{n}(-1)^{n+r} \epsilon(r) q^{n^{2}+2 n-r(r+1) / 2}
\end{aligned}
$$


where $\epsilon(r)=1$ if $r \equiv 1,2(\bmod 4)$ and $\epsilon(r)=0$ otherwise. We recall $(1.9)$ and note that

$$
\frac{(q ; q)_{\infty}}{(-q ; q)_{\infty}}=\frac{J_{1}^{2}}{J_{2}}=1+2 \sum_{n=1}^{\infty}(-1)^{n} q^{n^{2}} \equiv 1 \quad(\bmod 2) .
$$

Therefore we have

$$
D_{0}(q) \equiv 2 \sum_{n=0}^{\infty} \sum_{r=0}^{n}(-1)^{n+r} \epsilon(r) q^{n^{2}+2 n-r(r+1) / 2} \quad(\bmod 4) .
$$

Let

$$
\sum_{n=0}^{\infty} d(n) q^{n}:=\sum_{n=0}^{\infty} \sum_{r=0}^{n}(-1)^{n+r} \epsilon(r) q^{n^{2}+2 n-r(r+1) / 2} .
$$

Now assume $p \not \equiv \pm 1(\bmod 8)$ is an odd prime, and let $n, k$ be integers where $\left(\frac{k}{p}\right)=1$. Suppose $m=p^{2} n+(p k+1) \delta_{p}$, where $\delta_{p}=\frac{1}{8}\left(7 p^{2}+1\right)$. Then

$$
8 m+7 \equiv p k \quad\left(\bmod p^{2}\right),
$$

which implies that $p \| 8 m+7$. Since $p \equiv 3,5(\bmod 8)$, Lemma 4.2 implies $d_{v}(m)=0$. But $d_{v}(m)=0$ implies $d(m)=0$ and

$$
N_{A}(m+1) \equiv v(m) \quad(\bmod 4)
$$

by 4.5 . Now

$$
m+1 \equiv(p k+1) \delta_{p}-8 \delta_{p}+1 \equiv(p k+1) \delta_{p} \quad\left(\bmod p^{2}\right) .
$$

So $N_{A}(m+1) \equiv 0(\bmod 4)$ by Theorem 3.3 and hence $v(m) \equiv 0(\bmod 4)$.

4.2. The Andrews' $\operatorname{spt}(n)$ function. By equation (1.6) and [8, Theorem 1.3] we have the following theorem.

Theorem 4.3. For each $n>0, N_{\psi}(n)$ is odd if and only if

$$
24 n-1=p^{4 a+1} m^{2},
$$

for some prime $p$, and some integers $a, m$ satisfying $(m, p)=1$ and $a \geq 0$.

Berndt and Chan [13, p.776] found a Hecke-Rogers identity for the sixth order mock theta function $\phi_{-}(q)$.

$$
\frac{J_{1}^{2}}{J_{2}} \phi_{-}(q)=\sum_{n=1}^{\infty} \sum_{m=1-n}^{n}(-1)^{n-1} q^{n(3 n-1)-2 m^{2}+m}\left(1-q^{2 n}\right) .
$$

We need a similar Hecke-Rogers identity for $\psi(q)$.

Andrews [5, Eq. (1.10)], Mortenson [35, Eq. (2.5)], Chen and Wang [21, Eq. (4.37)] have found Hecke-Rogers series for $\psi(q)$. The second author [25] has shown how to express each of Ramanujan's third order mock theta functions in terms of Hecke-Rogers series. We need a new additional Hecke-Rogers series for $\psi(q)$. 
Using Jacobi's triple product identity 1.10 we find that

$$
\sum_{n=-\infty}^{\infty}(-1)^{n} q^{n(n+1) / 2+b n}=0
$$

for any integer $b$. Similarly for any integer $b$ we have

$$
\sum_{n=-\infty}^{\infty}(-1)^{n} q^{n(3 n+1) / 2+b n}=\left\{\begin{array}{lll}
(-1)^{b} q^{-b(b+1) / 2}(q)_{\infty}, & \text { if } b \equiv 0 & (\bmod 3), \\
0, & \text { if } b \equiv 1 & (\bmod 3), \\
(-1)^{b-1} q^{-b(b+1) / 2}(q)_{\infty}, & \text { if } b \equiv 2 & (\bmod 3)
\end{array}\right.
$$

See [12, p.99].

Lemma 4.4. We have

$$
\frac{J_{1}^{2}}{J_{2}} \psi(q)=\sum_{n=1}^{\infty} \sum_{m=1-n}^{n}(-1)^{m-1} q^{n(3 n-1)-2 m^{2}+m}\left(1-q^{2 n}\right) .
$$

Remark. We note that this Hecke-Rogers series for $\psi(q)$ may be deduced from a general theorem of Bradley-Thrush [19]. In fact a two-parameter generalization may be obtained from letting $k=2, p=q^{6}$ and $x=q^{-2}$ in [19, Theorem 7.3]. Our proof is different. This method will be used to obtain a new Hecke-Rogers series for $U(q)$, the generating function for strongly unimodal sequences. See Lemma 4.17 below.

Proof. From [21, Eq. (4.37)] we have

$$
J_{1} \psi(q)=\sum_{n=1}^{\infty} \sum_{r=1}^{n}(-1)^{n-1} q^{2 n^{2}-n-r(r-1) / 2}\left(1-q^{2 n}\right) .
$$

We note that (4.10) also follows from [31, $\mathrm{Eq}(1.3), \mathrm{Eq}(1.5)]$. We define

$$
A_{k}:=\sum_{n=1}^{\infty} \sum_{r=1}^{n}(-1)^{n-1} q^{2 n^{2}-n-(r-k)(r-k-1) / 2}\left(1-q^{2 n}\right),
$$

so that $A_{0}=J_{1} \psi(q)$. Using (4.8) we derive a recurrence relation for $A_{k}$.

$$
\begin{aligned}
& A_{k}+A_{-k}-\left(A_{k-1}+A_{1-k}\right) \\
& =\sum_{n=1}^{\infty} \sum_{r=1}^{n}(-1)^{n-1}\left(q^{2 n^{2}-n-(r-k)(r-k-1) / 2}+q^{2 n^{2}-n-(r+k)(r+k-1) / 2}\right)\left(1-q^{2 n}\right) \\
& -\sum_{n=1}^{\infty} \sum_{r=1}^{n}(-1)^{n-1}\left(q^{2 n^{2}-n-(r-k+1)(r-k) / 2}+q^{2 n^{2}-n-(r+k-1)(r+k-2) / 2}\right)\left(1-q^{2 n}\right) \\
& =\sum_{n=1}^{\infty}(-1)^{n}\left(q^{2 n^{2}-n-(n-k)(n-k+1) / 2}-q^{2 n^{2}-n-(n+k)(n+k-1) / 2}\right)\left(1-q^{2 n}\right)
\end{aligned}
$$




$$
\begin{aligned}
& =\sum_{n=-\infty}^{\infty}(-1)^{n} q^{n(3 n+1) / 2+n(1-k)-k(k-1) / 2}-\sum_{n=-\infty}^{\infty}(-1)^{n} q^{n(3 n+1) / 2+n k-k(k-1) / 2} \\
& =-J_{1} a_{k}
\end{aligned}
$$

where

$$
\begin{aligned}
& a_{3 r}:=(-1)^{r} q^{-6 r^{2}+r}, \\
& a_{3 r+1}:=(-1)^{r-1} q^{-6 r^{2}-r}, \\
& a_{3 r+2}:=(-1)^{r-1}\left(q^{-6 r^{2}-5 r-1}+q^{-6 r^{2}-7 r-2}\right) .
\end{aligned}
$$

Therefore for $k>0$ we have

$$
A_{k}+A_{-k}=2 A_{0}-J_{1} \sum_{r=1}^{k} a_{r}
$$

By (4.7) we find that

$$
\sum_{k=-\infty}^{\infty}(-1)^{k} A_{k} q^{k^{2}}=\sum_{n=1}^{\infty} \sum_{r=1}^{n}(-1)^{n-1} q^{2 n^{2}-n-r(r-1) / 2}\left(1-q^{2 n}\right) \sum_{k=-\infty}^{\infty}(-1)^{k} q^{k(k-1) / 2+r k}=0 .
$$

By 4.11

$$
\sum_{k=-\infty}^{\infty}(-1)^{k} A_{k} q^{k^{2}}=\frac{J_{1}^{2}}{J_{2}} A_{0}-J_{1} \sum_{k=1}^{\infty} \sum_{r=1}^{k}(-1)^{k} q^{k^{2}} a_{r}
$$

By (4.12) and 4.13) we have

$$
\frac{J_{1}^{2}}{J_{2}} A_{0}=J_{1} \sum_{k=1}^{\infty} \sum_{r=1}^{k}(-1)^{k} q^{k^{2}} a_{r}
$$

Let $G(r, k):=(-1)^{k} q^{k^{2}} a_{r}$ and $F(m, n):=\tilde{s g}(n)(-1)^{m-1} q^{n(3 n-1)-2 m^{2}+m}$, where $\tilde{s g}(n)=1$ for $n>0, \tilde{s g}(0)=0$ and $\tilde{s g}(n)=-1$ for $n<0$. It is easy to check that

$$
G(3 s+t, k)= \begin{cases}F(3 s-k, 2 s-k), & \text { if } t=0, \\ F(k-3 s, k-2 s), & \text { if } t=1, \\ F(3 s+2-k, k-2 s-1)+F(k-3 s-1,2 s-k+1) & \text { if } t=2,\end{cases}
$$

assuming $0<3 s+t \leq k$. We find that

$$
\sum_{k=1}^{\infty} \sum_{0<3 s \leq k} G(3 s, k)=\sum_{k=1}^{\infty} \sum_{0 \leq 3 s \leq k} F(3 s-k, 2 s-k)=\sum_{n=-\infty}^{-1} \sum_{m=1+n}^{0} F(m, n) .
$$


Similarly we find that

$$
\sum_{k=1}^{\infty} \sum_{0<3 s+1 \leq k} G(3 s+1, k)=\sum_{n=1}^{\infty} \sum_{m=1}^{n} F(m, n),
$$

and

$$
\sum_{k=1}^{\infty} \sum_{0<3 s+2 \leq k} G(3 s+2, k)=\left(\sum_{n=1}^{\infty} \sum_{m=1-n}^{0}+\sum_{n=-\infty}^{-1} \sum_{m=1}^{-n}\right) F(m, n) .
$$

Hence by 4.14 - 4.17

$$
\begin{aligned}
\frac{J_{1}^{2}}{J_{2}} \psi(q) & =\sum_{k=1}^{\infty} \sum_{r=1}^{k} G(r, k)=\sum_{n=-\infty}^{\infty} \sum_{m=1-|n|}^{|n|} F(m, n) \\
& =\sum_{n=1}^{\infty} \sum_{m=1-n}^{n}(-1)^{m-1} q^{n(3 n-1)-2 m^{2}+m}\left(1-q^{2 n}\right) .
\end{aligned}
$$

We define the following three series

$$
\begin{aligned}
& D_{0}(q):=\sum_{n=1}^{\infty} d_{0}(n) q^{n}:=\sum_{n=-\infty}^{\infty} \sum_{1-|n| \leq m \leq|n|} q^{n(3 n-1)-m(2 m-1)}, \\
& D_{1}(q):=\sum_{n=1}^{\infty} d_{1}(n) q^{n}:=\sum_{n=-\infty}^{\infty} \sum_{1-|n| \leq m \leq|n|}(1-\epsilon(m, n)) q^{n(3 n-1)-m(2 m-1)}, \\
& D_{2}(q):=\sum_{n=1}^{\infty} d_{2}(n) q^{n}:=\sum_{n=-\infty}^{\infty} \sum_{1-|n| \leq m \leq|n|} \epsilon(m, n) q^{n(3 n-1)-m(2 m-1)},
\end{aligned}
$$

where $\epsilon(m, n)=1$ if $m \equiv n(\bmod 2)$ and $\epsilon(m, n)=0$ otherwise. Clearly $D_{0}(q)=D_{1}(q)+$ $D_{2}(q)$.

Lemma 4.5. We have

$$
\phi_{-}(q)-\psi(q) \equiv 2 D_{1}(q) \quad(\bmod 4) .
$$

Proof. By 4.6 and 4.9

$$
\begin{aligned}
\phi_{-}(q)-\psi(q) & =\frac{J_{2}}{J_{1}^{2}} \sum_{n=1}^{\infty} \sum_{m=1-n}^{n}\left((-1)^{n-1}-(-1)^{m-1}\right) q^{n(3 n-1)-2 m^{2}+m}\left(1-q^{2 n}\right) \\
& \equiv 2 \sum_{n=1}^{\infty} \sum_{j=1-n}^{n} \frac{(-1)^{n-1}-(-1)^{m-1}}{2} q^{n(3 n-1)-2 m^{2}+m}\left(1+q^{2 n}\right)
\end{aligned}
$$




$$
\begin{aligned}
& \equiv 2 \sum_{n=-\infty}^{\infty} \sum_{1-|n| \leq m \leq|n|}(1-\epsilon(m, n)) q^{n(3 n-1)-2 m^{2}+m} \\
& \equiv 2 D_{1}(q) \quad(\bmod 4) .
\end{aligned}
$$

We note that Lemma 4.5 implies that $N_{\phi_{-}}(n)$ and $N_{\psi}(n)$ have the same parity. This is confirmed by Theorem 3.9(1) and Theorem 4.3. The following two lemmas are related to solutions of the Pell equation

$$
u^{2}-6 v^{2}=m
$$

where $u, v, m$ are integers. Following [7] we say two solutions $(u, v)$ and $\left(u^{\prime}, v^{\prime}\right)$ are equivalent if

$$
u^{\prime}+v^{\prime} \sqrt{6}= \pm(5+2 \sqrt{6})^{r}(u+v \sqrt{6}),
$$

for some integer $r$. By [7, Lemma 3], if $m>0$, then each equivalence class of solutions of (4.20) contains a unique $(u, v)$ with $u>0$ and

$$
-\frac{1}{3} u<v \leq \frac{1}{3} u
$$

We define $H_{\sqrt{6}}(m)$ to be the number of inequivalent solutions to 4.20 . By 4.22 we have

$$
\sum_{m=1}^{\infty} H_{\sqrt{6}}(m) q^{m}=\sum_{u=1}^{\infty} \sum_{-\frac{1}{3} u<v \leq \frac{1}{3} u} q^{u^{2}-6 v^{2}} .
$$

Lovejoy [32] has calculated $H_{\sqrt{6}}(m)$. Wang [42, Lemma 2.7] extended this to negative $m$.

Lemma 4.6 (Lovejoy [32, Theorem 1.3]). Let $m$ have prime factorization

$$
m=2^{a} 3^{b} \prod_{i=1}^{\ell} p_{i}^{e_{i}} \prod_{j=1}^{n} q_{j}^{f_{j}} \prod_{k=1}^{t} r_{k}^{g_{k}}
$$

where the $p_{i} \equiv \pm 7, \pm 11(\bmod 24)$, the $q_{j} \equiv 1,19(\bmod 24)$, and the $r_{k} \equiv 5,23(\bmod 24)$. Then

$$
H_{\sqrt{6}}(m)= \begin{cases}0, & \text { if some } e_{i} \text { is odd or } a+\sum g_{k} \text { is odd } \\ \prod_{j=1}^{n}\left(f_{j}+1\right) \prod_{k=1}^{t}\left(g_{k}+1\right), & \text { otherwise. }\end{cases}
$$

Corollary 4.7. Let $24 n-1$ have the prime factorization

$$
24 n-1=\prod_{i=1}^{k} p_{i}^{a_{i}} \prod_{j=1}^{l} q_{j}^{b_{j}}
$$


where $p_{i} \equiv 1,5,19,23(\bmod 24)$ and $q_{j} \equiv 7,11,13,17(\bmod 24)$. If some $b_{j}$ is odd then $d_{0}(n)=0$, otherwise we have

$$
d_{0}(n)=\frac{1}{2} \prod_{i=1}^{k}\left(a_{i}+1\right) .
$$

Proof. We note $u^{2}-6 v^{2} \equiv-2(\bmod 48)$ if and only if $u \equiv \pm 2(\bmod 12)$ and $v$ is odd. Thus by 4.23 we find that

$$
\begin{aligned}
\sum_{n=1}^{\infty} H_{\sqrt{6}}(48 n-2) & =\sum_{n=1}^{\infty}\left(\sum_{-2 n<m \leq 2 n} q^{(12 n-2)^{2}-6(2 m-1)^{2}}+\sum_{-2 n<m \leq 2 n} q^{(12 n+2)^{2}-6(2 m-1)^{2}}\right) \\
& =2 \sum_{n=1}^{\infty}\left(\sum_{-n<m \leq n} q^{(12 n-2)^{2}-6(4 m-1)^{2}}+\sum_{-n<m \leq n} q^{(12 n+2)^{2}-6(4 m-1)^{2}}\right) \\
& =2 \sum_{n=1}^{\infty} d_{0}(n) q^{48 n-2} .
\end{aligned}
$$

If some $b_{j}$ is odd then by Lemma $4.6 H_{\sqrt{6}}(48 n-2)=0$ and $d_{0}(n)=0$. We assume all the $b_{j}$ are even and note that $p^{2} \equiv 1(\bmod 24)$ for $(p, 6)=1$. Next we show that the number of primes congruent to 5 or 23 (counted with multiplicity) in the factorization of $48 n-2$ is odd. If it is even then the product of these primes is either 1 or $5 \cdot 23 \equiv 19(\bmod 24)$. But this would imply that $24 n-1$ is either 1 or $19(\bmod 24)$, which is a contradiction. The result follows from 4.25 and Lemma 4.6.

We also need Andrews, Dyson and Hickerson's [7] results for

$$
\sum_{n=1}^{\infty} S^{*}(n) q^{n}=\sum_{n=1}^{\infty} \frac{(-1)^{n} q^{n^{2}}}{\left(q ; q^{2}\right)_{n}} .
$$

Lemma 4.8. For $n \geq 1$ let $s(n):=d_{1}(n)-d_{2}(n)$ and

$$
24 n-1=\prod_{i=1}^{k} p_{i}^{a_{i}} \prod_{j=1}^{l} q_{j}^{b_{j}}
$$

be the prime factorization of $24 n-1$, where the $p_{i} \equiv \pm 1(\bmod 24)$, and the $q_{j} \equiv \pm 5, \pm 7, \pm 11$ $(\bmod 24)$. If some $b_{j} \equiv 1(\bmod 2)$ then $s(n)=0$, otherwise we have

$$
|s(n)|=\frac{1}{2} \prod_{i=1}^{k}\left(a_{i}+1\right) .
$$

Proof. By [7, Eq.(5.2)] we find that

$$
\sum_{n=1}^{\infty} S^{*}(n) q^{n}=\sum_{n=1}^{\infty} \sum_{j=0}^{2 n-1}(-1)^{n} q^{n(3 n-1)-j(j+1) / 2}\left(1+q^{n}\right)
$$




$$
=\sum_{n=1}^{\infty} \sum_{m=-n+1}^{n}(-1)^{n} q^{n(3 n-1)-m(2 m-1)}\left(1+q^{n}\right)=\sum_{n=-\infty}^{\infty} \sum_{m=-|n|+1}^{|n|}(-1)^{n} q^{n(3 n-1)-m(2 m-1)} .
$$

After replacing $q$ by $-q$ we have

$$
\sum_{n=1}^{\infty}(-1)^{n+1} S^{*}(n) q^{n}=\sum_{n=-\infty}^{\infty} \sum_{m=-|n|+1}^{|n|}(-1)^{m+n+1} q^{n(3 n-1)-m(2 m-1)}=\sum_{n=1}^{\infty} s(n) q^{n} .
$$

Hence

$$
|s(n)|=\left|S^{*}(n)\right|
$$

By [7, Theorem 5] we have

$$
S^{*}(n)=\frac{1}{2} T(1-24 n),
$$

where $T(m)$ is the excess of the number of solutions of 4.20$)$ satisfying $u+3 v \equiv \pm 1(\bmod 12)$ over the number satisfying $u+3 v \equiv \pm 5(\bmod 12)$. The result then follows from [7, Theorem $3]$ which gives a formula for $T(m)$ in terms of the prime factorisation of $m$ when $m \equiv 1$ $(\bmod 6)$.

We can determine the difference $D_{1}(q)$ by Lemma 4.7 and Lemma 4.8. First we consider the case when is square-free.

Lemma 4.9. Let $n$ be an integer such that $N_{\psi}(n)$ is even and $24 n-1$ is square-free. Then $d_{1}(n)$ is odd if and only if

$$
24 n-1=p_{1} p_{2},
$$

where $p_{1}$ and $p_{2}$ are primes for which $\left\{p_{1}, p_{2}\right\} \equiv\{5,19\}(\bmod 24)$.

Proof. Suppose $N_{\psi}(n)$ is even where $24 n-1$ is a square-free positive integer. Let

$$
24 n-1=\prod_{i=1}^{k} p_{i}
$$

be the prime factorization of $24 n-1$. If $k=1$ then Theorem 4.3 implies that $N_{\psi}(n)$ is odd which is a contradiction. Hence either $k=2$ or $k>2$. If $k>2$ then Lemmas 4.7 and 4.8 imply that $d_{0}(n) \equiv s(n) \equiv 0(\bmod 4)$ and

$$
2 d_{1}(n)=d_{0}(n)+s(n) \equiv 0 \quad(\bmod 4) \quad \text { and } \quad d_{1} \equiv 0 \quad(\bmod 2) .
$$

Now suppose $k=2$, so that $24 n-1$ is a product of two primes $p_{1}$ and $p_{2}$. We note that $p_{1} \equiv-p_{2}(\bmod 24)$. There are three cases.

Case 1: $\left\{p_{1}, p_{2}\right\} \equiv\{1,23\}(\bmod 24)$. Lemmas 4.7 and 4.8 imply that

$$
2 d_{1}(n)=d_{0}(n)+s(n)=2 \pm 2 \equiv 0 \quad(\bmod 4), \quad \text { and } \quad d_{1} \equiv 0 \quad(\bmod 2) .
$$

Case 2: $\left\{p_{1}, p_{2}\right\} \equiv\{5,19\}(\bmod 24)$. Lemmas 4.7 and 4.8 imply that

$$
2 d_{1}(n)=d_{0}(n)+s(n)=2+0=2, \quad \text { and } \quad d_{1}=1 .
$$


Case 3: $\left\{p_{1}, p_{2}\right\} \equiv\{7,17\}(\bmod 24)$. Lemmas 4.7 and 4.8 imply that

$$
2 d_{1}(n)=d_{0}(n)+s(n)=0+0=0, \quad \text { and } \quad d_{1}=0 .
$$

Case 4: $\left\{p_{1}, p_{2}\right\} \equiv\{11,13\}(\bmod 24)$. Lemmas 4.7 and 4.8 imply that

$$
2 d_{1}(n)=d_{0}(n)+s(n)=0+0=0, \quad \text { and } \quad d_{1}=0 .
$$

Hence we see that $d_{1}(n)$ is odd if and only if $\left\{p_{1}, p_{2}\right\} \equiv\{5,19\}(\bmod 24)$.

To extend Lemma 4.9 to the case when $24 n-1$ is not square-free we need an analog of Lemma 2.4. Fortunately we have the following result.

Theorem 4.10 ([24, Theorem 1.3(i)]). If $\ell \geq 5$ is prime then

$$
\operatorname{spt}\left(\ell^{2} n-s_{\ell}\right)+\left(\frac{3}{\ell}\right)\left(\frac{1-24 n}{\ell}\right) \operatorname{spt}(n)+\ell \operatorname{spt}\left(\frac{n+s_{\ell}}{\ell^{2}}\right) \equiv\left(\frac{3}{\ell}\right)(1+\ell) \operatorname{spt}(n) \quad(\bmod 72),
$$

where $s_{\ell}=\frac{1}{24}\left(\ell^{2}-1\right)$.

By 1.6 we have the following corollary.

Corollary 4.11. If $\ell \geq 5$ is prime then

$$
\begin{aligned}
& N_{\psi}\left(\ell^{2} n-s_{\ell}\right)+(-1)^{s_{\ell}}\left(\frac{3}{\ell}\right)\left(\frac{1-24 n}{\ell}\right) N_{\psi}(n)+\ell N_{\psi}\left(\frac{n+s_{\ell}}{\ell^{2}}\right) \\
& \equiv(-1)^{s_{\ell}}\left(\frac{3}{\ell}\right)(1+\ell) N_{\psi}(n) \quad(\bmod 4) .
\end{aligned}
$$

Following [11, p.353] we use the standard practice of rewriting an arithmetic function in terms of $24 n-1$. We write

$$
\tilde{N}_{\psi}(m)= \begin{cases}N_{\psi}(n), & \text { if } m=24 n-1 \\ 0, & \text { if } m<23 \text { or } m \not \equiv-1 \quad(\bmod 24) \text { or } m \text { is non-integral. }\end{cases}
$$

We rewrite Corollary 4.11 in terms $\tilde{N}_{\psi}$ and derive some congruence properties.

Lemma 4.12. Let $\ell \geq 5$ be prime. Then

$$
\tilde{N}_{\psi}\left(\ell^{2} n\right)+(-1)^{s_{\ell}}\left(\frac{-3 n}{\ell}\right) \tilde{N}_{\psi}(n)+\ell \tilde{N}_{\psi}\left(\frac{n}{\ell^{2}}\right) \equiv(-1)^{s_{\ell}}\left(\frac{3}{\ell}\right)(1+\ell) \tilde{N}_{\psi}(n) \quad(\bmod 4)
$$

(ii) If $(\ell, n)=1$ and $\tilde{N}_{\psi}(n)$ is even then

(a) $\tilde{N}_{\psi}\left(\ell^{2} n\right) \equiv \tilde{N}_{\psi}(n)(\bmod 4)$,

(b) $\tilde{N}_{\psi}\left(\ell^{3} n\right) \equiv 0(\bmod 4)$.

$$
\tilde{N}_{\psi}\left(\ell^{4} n\right) \equiv \tilde{N}_{\psi}(n) \quad(\bmod 4)
$$


Proof. (i). The congruence (4.28) follows immediately from (4.27).

(ii). Suppose $(n, \ell)=1$ and $\tilde{N}_{\psi}(n)$ is even. Since $\ell$ is odd we have $(1+\ell) \tilde{N}_{\psi}(n) \equiv 0$ $(\bmod 4)$, and $\tilde{N}_{\psi}\left(n / \ell^{s}\right)=0$ since $(n, \ell)=1$. The congruence 4.28 implies that

$$
\tilde{N}_{\psi}\left(\ell^{2} n\right) \equiv \tilde{N}_{\psi}(n) \quad(\bmod 4)
$$

Replacing $n$ by $\ell n$ in 4.28 gives

$$
\tilde{N}_{\psi}\left(\ell^{3} n\right) \equiv(-1)^{s \ell}\left(\frac{3}{\ell}\right)(1+\ell) \tilde{N}_{\psi}(\ell n) \quad(\bmod 4) .
$$

Either $\tilde{N}_{\psi}(\ell n)$ is even or $\tilde{N}_{\psi}(\ell n)$ is odd and $\ell \equiv-1(\bmod 24)$. In both cases we have $(1+\ell) \tilde{N}_{\psi}(\ell n) \equiv 0(\bmod 4)$ and

$$
\tilde{N}_{\psi}\left(\ell^{3} n\right) \equiv 0 \quad(\bmod 4) .
$$

(iii). Replacing $n$ by $\ell^{2} n$ in (4.27) we have

$$
\tilde{N}_{\psi}\left(\ell^{4} n\right) \equiv-\ell \tilde{N}_{\psi}(n)+(-1)^{s_{\ell}}\left(\frac{3}{\ell}\right)(1+\ell) \tilde{N}_{\psi}\left(\ell^{2} n\right) \quad(\bmod 4) .
$$

There are three cases.

Case 1: $\ell \equiv 3(\bmod 4)$. From 4.29 we have

$$
\tilde{N}_{\psi}\left(\ell^{4} n\right) \equiv \tilde{N}_{\psi}(n) \quad(\bmod 4)
$$

since $-\ell \equiv 1(\bmod 4)$ and $(1+\ell) \equiv 0(\bmod 4)$.

Case $2: \ell \equiv 1(\bmod 4)$ and $\tilde{N}_{\psi}\left(\ell^{2} n\right)$ is odd. Since $\ell \not \equiv 23(\bmod 24)$ Theorem 4.3 implies that $\tilde{N}_{\psi}(n)$ is also odd, and from 4.29 we have

$$
\begin{aligned}
\tilde{N}_{\psi}\left(\ell^{4} n\right) & \equiv \tilde{N}_{\psi}(n)-(1+\ell)\left(\tilde{N}_{\psi}(n)-(-1)^{s_{\ell}}\left(\frac{3}{\ell}\right) \tilde{N}_{\psi}\left(\ell^{2} n\right)\right) \quad(\bmod 4) \\
& \equiv \tilde{N}_{\psi}(n) \quad(\bmod 4) .
\end{aligned}
$$

Case 3: $\ell \equiv 1(\bmod 4)$ and $\tilde{N}_{\psi}\left(\ell^{2} n\right)$ is even. Again since $\ell \not \equiv 23(\bmod 24)$ Theorem 4.3 implies that $\tilde{N}_{\psi}(n)$ is also even and from 4.29 we see that

$$
\tilde{N}_{\psi}\left(\ell^{4} n\right) \equiv \tilde{N}_{\psi}(n) \quad(\bmod 4)
$$

This completes the proof of (iii) in all cases.

Theorem 4.13. For $n>0$ be an integer, $N_{\psi}(n) \equiv 2(\bmod 4)$ if and only if $24 n-1$ has the form

$$
24 n-1=p_{1}^{4 a+1} p_{2}^{4 b+1} m^{2},
$$


where $p_{1}$ and $p_{2}$ are primes such that $\left(\frac{p_{1}}{p_{2}}\right)=-\varepsilon\left(p_{2}\right)$ for $\varepsilon(p)=-1$ if $p \equiv \pm 5(\bmod 24)$ and $\varepsilon(p)=1$ otherwise, $\left(m, p_{1} p_{2}\right)=1$ and $a, b \geq 0$ are integers.

Proof. Suppose $n>0$. First we prove the result when $24 n-1$ is square-free. By Lemma 4.5

$$
N_{\phi_{-}}(n)-N_{\psi}(n) \equiv 2 d_{1}(n) \quad(\bmod 4) .
$$

We assume $24 n-1$ is square-free and $N_{\psi}(n) \equiv 2(\bmod 4)$. The congruence 4.30$)$ implies that $N_{\phi_{-}}(n) \equiv 0$ or $2(\bmod 4)$.

Case 1: $N_{\phi_{-}}(n) \equiv 0(\bmod 4)$. Therefore $d_{1}(n)$ is odd and Lemma 4.9 implies that $24 n-1=$ $p_{1} p_{2}$ for primes $p_{1}, p_{2}$ satisfying $\left\{p_{1}, p_{2}\right\} \equiv\{5,19\}(\bmod 24)$. Theorem 3.9 implies that $\left(\frac{p_{1}}{p_{2}}\right)=1=-\varepsilon\left(p_{2}\right)$.

Case 2: $N_{\phi_{-}}(n) \equiv 2(\bmod 4)$. By Theorem 3.9 there are primes $p_{1}, p_{2}$ satisfying $\left(\frac{p_{1}}{p_{2}}\right)=-1$ and $24 n-1=p_{1} p_{2}$. By 4.30 $d_{1}(n)$ is even and Lemma 4.9 implies that $p_{1}, p_{2} \not \equiv \pm 5$ $(\bmod 24)$, so that $\left(\frac{p_{1}}{p_{2}}\right)=-\varepsilon\left(p_{2}\right)$.

Similarly we can show the converse that if $24 n-1=p_{1} p_{2}$ where $p_{1}$ and $p_{2}$ are primes satisfying $\left(\frac{p_{1}}{p_{2}}\right)=-\varepsilon\left(p_{2}\right)$, then $N_{\psi}(n) \equiv 2(\bmod 4)$. This completes the proof of the result when $24 n-1$ is square-free.

We now consider the general case. For this part we first assume that $N_{\psi}(n) \equiv 2(\bmod 4)$. We let $M=24 n-1$ so that $\tilde{N}_{\psi}(M)=N_{\psi}(n)$ and $\tilde{N}_{\psi}(M) \equiv 2(\bmod 4)$. We write the prime factorization of $M$ as

$$
M=\prod_{i=1}^{k} p_{i}^{4 a_{i}+r_{i}}
$$

where the $a_{i}, r_{i}$ are integers satisfying $0 \leq r_{i} \leq 3$. Then by Lemma 4.12 (iii) we have

$$
\tilde{N}_{\psi}(M) \equiv \tilde{N}_{\psi}\left(M^{\prime}\right) \equiv 2(\bmod 4)
$$

where

$$
M^{\prime}=\prod_{i=1}^{k} p_{i}^{r_{i}} \equiv-1 \quad(\bmod 24) .
$$

Lemma 4.12(ii)(b) implies that none of the $r_{i}$ are equal to 3. Lemma 4.12(ii)(a) implies that

$$
\tilde{N}_{\psi}(M) \equiv \tilde{N}_{\psi}\left(M^{\prime \prime}\right) \equiv 2 \quad(\bmod 4),
$$

where

$$
M^{\prime \prime}=\prod_{j \in J} p_{i} \equiv-1 \quad(\bmod 24),
$$

and $J$ is the set of $j$ for which $r_{j}=1$. Here $M^{\prime \prime}$ is square-free. Therefore $J$ is a set of two primes. Without loss of generality we may assume these two primes are $p_{1}$ and $p_{2}$ where $\left(\frac{p_{1}}{p_{2}}\right)=-\varepsilon\left(p_{2}\right)$. Hence

$$
24 n-1=M=p_{1}^{4 a_{1}+1} p_{2}^{4 a_{2}+1} m^{2},
$$


where $\left(m, p_{1} p_{2}\right)=1$ and $24 n-1$ has the desired form.

Finally we assume

$$
M=24 n-1=p_{1}^{4 a+1} p_{2}^{4 b+1} m^{2},
$$

where $p_{1}$ and $p_{2}$ are primes such that $\left(\frac{p_{1}}{p_{2}}\right)=-\varepsilon\left(p_{2}\right)$. Arguing as before and using Lemma 4.12 (ii),(iii) we have

$$
N_{\psi}(n)=\tilde{N}_{\psi}(M) \equiv \tilde{N}_{\psi}\left(p_{1} p_{2}\right) \quad(\bmod 4),
$$

where $M \equiv p_{1} p_{2} \equiv-1(\bmod 24)$. Since $p_{1} p_{2}$ is square-free, satisfies $\left(\frac{p_{1}}{p_{2}}\right)=-\varepsilon\left(p_{2}\right)$ and we have proved the square-free case $\tilde{N}_{\psi}\left(p_{1} p_{2}\right) \equiv 2(\bmod 4)$, the result

$$
N_{\psi}(n) \equiv 2 \quad(\bmod 4)
$$

follows.

We are now ready to complete the proof of Conjecture 1.3 and related congruences for $\psi(q)$.

Theorem 4.14. Let $p>3$ be a prime where $p \not \equiv 23(\bmod 24)$. Suppose $24 \delta_{p} \equiv 1\left(\bmod p^{2}\right)$, $k, n \in \mathbb{Z}$ and $\left(\frac{k}{p}\right)=\varepsilon(p)$ where $\varepsilon(p)=-1$ if $p \equiv \pm 5(\bmod 24)$ and $\varepsilon(p)=1$ otherwise. Then

$$
\begin{array}{ll}
N_{\psi}\left(p^{2} n+(p k+1) \delta_{p}\right) \equiv 0 & (\bmod 4), \\
\operatorname{spt}\left(p^{2} n+(p k+1) \delta_{p}\right) \equiv 0 & (\bmod 4) .
\end{array}
$$

Remark. We have rewritten the statement of Conjecture 1.3 in an equivalent form. The equivalence follows from the observations that $\left(\frac{6}{p}\right)=1$ for $p \equiv 1, \pm 5(\bmod 24)$ and $\left(\frac{6}{p}\right)=-1$ for $p \equiv \pm 7, \pm 11(\bmod 24)$. We note that a weak version of the $\bmod 4$ congruences for $N_{\psi}(n)$ were conjectured by Bryson, Ono Pitman and Rhoades [18, Eq.(1.7), Conjecture 1.6]. We discuss this further in Section 4.3 .

Proof. Assume $p>3$ is prime, $p \not \equiv 23(\bmod 24), 24 \delta_{p} \equiv 1\left(\bmod p^{2}\right), k, n \in \mathbb{Z}$ and $\left(\frac{k}{p}\right)=$ $\varepsilon(p)$. We let $m=p^{2} n+(p k+1) \delta_{p}$, so that

$$
24 m-1 \equiv p k \quad\left(\bmod p^{2}\right) .
$$

By Theorem 4.3 we see that $N_{\psi}(m)$ is even since $24 m-1$ and $p \not \equiv 23(\bmod 24)$. Now suppose that $N_{\psi}(m) \equiv 2(\bmod 4)$. By Theorem $4.13 N_{\psi}(m) \equiv 2(\bmod 4)$ if and only if $24 m-1$ has the form

$$
24 m-1=p^{1} q^{4 b+1} t^{2}
$$


where $q$ is a prime satisfying $\left(\frac{p}{q}\right)=-\varepsilon(q)(p q, t)=1$, and $b, t>0$ are integers. Either $p$ or $q \equiv 1(\bmod 4)$ so by quadratic reciprocity $\left(\frac{q}{p}\right)=\left(\frac{p}{q}\right)=-\varepsilon(q)$. From 4.33 we have

$$
k \equiv q^{4 b+1} t^{2} \quad(\bmod p) .
$$

But this implies that

$$
\left(\frac{q}{p}\right)=\left(\frac{k}{p}\right)=\varepsilon(q),
$$

which is a contradiction. Hence $N_{\psi}(m) \not \equiv 2(\bmod 4)$ and

$$
N_{\psi}(m) \equiv 0 \quad(\bmod 4),
$$

which is 4.31). Finally the result 4.32 holds by (1.6).

4.3. Strongly unimodal sequences. In this section we prove Bryson, Ono, Pitman and Rhoades's Conjecture 1.1. This conjecture has three parts. In this section we will prove the first part, congruence (1.2). The third part, congruence (1.4), follows from Theorem 4.14. The second part, congruence (1.3), will follow from the first and third parts. As noted in Section 1 we have

$$
\mathcal{U}( \pm i ; q)=\psi(q)=\sum_{n=1}^{\infty} N_{\psi}(n) q^{n}=\sum_{n=1}^{\infty} \frac{q^{n^{2}}}{\left(q ; q^{2}\right)_{n}}
$$

See [18, p.16064]. Also define $U(q)$ by

$$
U(q):=\mathcal{U}(1 ; q)=\sum_{n=1}^{\infty} u(n) q^{n} .
$$

Letting $z=i$ in $(1.1)$ we have

$$
u(0,4 ; n)-u(2,4, n)=N_{\psi}(n), \quad \text { and } \quad u(1,4 ; n)=u(3,4 ; n) .
$$

Hence

$$
u(n)=u(0,4 ; n)+2 u(1,4 ; n)+u(2,4 ; n),
$$

and

$$
u(n)+N_{\psi}(n)=2(u(0,4 ; n)+u(1,4 ; n)) .
$$

From (4.36) it is clear that (1.4) follows from Theorem 4.14. Equations 4.36-4.38) together with Theorem 4.14 show how (1.3) follows from $(1.2)$ and (1.4). Here we assume that $\ell \equiv 7,11,13,17(\bmod 24)$ is prime and $\left(\frac{k}{\ell}\right)=-1$.

We point out that there is a stronger result for Bryson, Ono, Pitman's Conjecture (1.4) that includes primes $\ell \equiv 1, \pm 5(\bmod 24)$. This follows easily from 4.31 and (4.36). We state this as a separate theorem. 
Theorem 4.15. Suppose $\ell>i$ s prime and $\ell \not \equiv 23(\bmod 24)$. Then for $n \geq 0$ we have

$$
u\left(0,4 ; \ell^{2} n+k l-s(\ell)\right) \equiv u\left(2,4 ; \ell^{2} n+k l-s(\ell)\right) \quad(\bmod 4),
$$

provided $\left(\frac{k}{\ell}\right)=\tilde{\varepsilon}(\ell)$ and $s(\ell)=\frac{1}{24}\left(\ell^{2}-1\right)$.

Remark. Here $\tilde{\varepsilon}(\ell)$ is defined in the statement of Conjecture 1.3; i.e. $\tilde{\varepsilon}(\ell)=1$ if $\ell \equiv 1$ $(\bmod 24)$ and -1 otherwise. For example let $\ell=457 \equiv 1(\bmod 24)$, and $k=21$. Then $\left(\frac{k}{\ell}\right)=\left(\frac{21}{457}\right)=1=\tilde{\varepsilon}(\ell), s(\ell)=8702$, and $k \ell-s(\ell)=895$. We have

$$
\begin{array}{rlrl}
u(0,4 ; 895) & =256203223294825619203431487908 \equiv 0 & & (\bmod 4) \\
u(1,4 ; 895)=u(3,4,895) & =256203223294825426775345978961 \equiv 1 & (\bmod 4) \\
u(2,4 ; 895) & =256203223294825234347260470016 \equiv 0 & (\bmod 4) \\
u(895) & =1024812893179301707101383915846 \equiv 2 & & (\bmod 4) .
\end{array}
$$

Hikami and Lovejoy [28, Theorem 4.1] found a Hecke-Rogers identity for the generating function $\mathcal{U}(z ; q)$;

$$
(1+z) \mathcal{U}(z ; q)=\frac{q}{(q ; q)_{\infty}}\left(\sum_{r, n \geq 0}-\sum_{r, n<0}\right)(-1)^{n} z^{-r} q^{n(3 n+5) / 2+2 n r+r(r+3) / 2} .
$$

See also [31, Eq.(1.3)]. Considering Lemma 4.4 and that $\psi(q)=\mathcal{U}(i ; q)$ it is reasonable to suspect that $\mathcal{U}(1 ; q)$ has a similar Hecke-Rogers identity.

Lemma 4.16. We have

$$
J_{1} U(q)=\sum_{n=1}^{\infty} \sum_{r=1}^{n}(-1)^{r-1} q^{2 n^{2}-n-r(r-1) / 2}\left(1+q^{2 n}\right) .
$$

Proof. We define

$$
Q(n, r):=n(3 n+5) / 2+2 n r+r(r+3) / 2+1
$$

and

$$
\mathcal{F}(z ; q)=\sum_{r=0}^{\infty} \sum_{n=0}^{\infty}(-1)^{n} z^{-r} q^{Q(n, r)}
$$

Thus 4.40 can be rewritten as

$$
(1+z) \mathcal{U}(z ; q)=\frac{1}{(q ; q)_{\infty}}\left(\mathcal{F}(z ; q)+z \mathcal{F}\left(z^{-1} ; q\right)\right)
$$

since

$$
Q(n, r)=Q(-n-1,-r-1) .
$$

We observe that

$$
Q(n-1, r-2 n+1)=r(r+1) / 2-n(n-1) / 2,
$$


so that

$$
\mathcal{F}(z ; q)=\sum_{n=1}^{\infty} \sum_{r=2 n-1}^{\infty}(-1)^{n+1} z^{2 n-r-1} q^{r(r+1) / 2-n(n-1) / 2} .
$$

Letting $z=1$ in 4.42 we find that

$$
\begin{aligned}
J_{1} U(q) & =J_{1} \mathcal{U}(1 ; q)=\mathcal{F}(1 ; q)=\sum_{n=1}^{\infty} \sum_{r=2 n-1}^{\infty}(-1)^{n+1} q^{r(r+1) / 2-n(n-1) / 2} \\
& =\sum_{r=1}^{\infty} \sum_{n=1}^{\lfloor(r+1) / 2\rfloor}(-1)^{n+1} q^{r(r+1) / 2-n(n-1) / 2} \\
& =\sum_{r=1}^{\infty} \sum_{n=1}^{r}(-1)^{n+1}\left(q^{r(2 r+1)-n(n-1) / 2}+q^{r(2 r-1)-n(n-1) / 2}\right),
\end{aligned}
$$

by replacing $r$ by $2 r$ and $2 r-1$ in the previous sum. The result (4.41) follows easily by interchanging $r$ and $n$.

The proof of the next lemma, which contains our new Hecke-Rogers identity for $U(q)$, is analogous to that of Lemma 4.4 .

Lemma 4.17. We have

$$
\frac{J_{1}^{2}}{J_{2}} U(q)=\sum_{n=1}^{\infty} \sum_{m=1-n}^{n} s g(m)(-1)^{n-1} q^{n(3 n-1)-2 m^{2}+m}\left(1+q^{2 n}\right),
$$

where $s g(m)=1$ if $m>0$ and $s g(m)=-1$ otherwise.

Proof. We define

$$
A_{k}:=\sum_{n=1}^{\infty} \sum_{r=1}^{n}(-1)^{r+k-1} q^{2 n^{2}-n-(r-k)(r-k-1) / 2}\left(1+q^{2 n}\right),
$$

so that $A_{0}=J_{1} U(q)$ by Lemma 4.16. Using $(1.10)$ and $(4.8)$ we derive a recurrence relation for $A_{k}$ :

$$
\begin{aligned}
& A_{k}+A_{-k}-\left(A_{k-1}+A_{1-k}\right) \\
& =\sum_{n=1}^{\infty} \sum_{r=1}^{n}(-1)^{r+k-1}\left(q^{2 n^{2}-n-(r-k)(r-k-1) / 2}+q^{2 n^{2}-n-(r+k)(r+k-1) / 2}\right)\left(1+q^{2 n}\right) \\
& -\sum_{n=1}^{\infty} \sum_{r=1}^{n}(-1)^{r+k}\left(q^{2 n^{2}-n-(r-k+1)(r-k) / 2}+q^{2 n^{2}-n-(r+k-1)(r+k-2) / 2}\right)\left(1+q^{2 n}\right) \\
& =(-1)^{k} \sum_{n=1}^{\infty}\left(q^{2 n^{2}-n-k(k-1) / 2}+q^{2 n^{2}-n-k(k-1) / 2}\right)\left(1+q^{2 n}\right)
\end{aligned}
$$




$$
\begin{aligned}
& -(-1)^{k} \sum_{n=1}^{\infty}(-1)^{n}\left(q^{2 n^{2}-n-(n-k)(n-k+1) / 2}+q^{2 n^{2}-n-(n+k)(n+k-1) / 2}\right)\left(1+q^{2 n}\right) \\
& =(-1)^{k}\left(2 \sum_{n=-\infty}^{\infty} q^{2 n^{2}-n-k(k-1) / 2}-\sum_{n=-\infty}^{\infty}(-1)^{n} q^{n(3 n+1) / 2+n(1-k)-k(k-1) / 2}\right. \\
& \left.-\sum_{n=-\infty}^{\infty}(-1)^{n} q^{n(3 n+1) / 2+n k-k(k-1) / 2}\right) \\
& =2 \frac{J_{2}^{2}}{J_{1}}(-1)^{k} q^{-k(k-1) / 2}-J_{1} a_{k} .
\end{aligned}
$$

where

$$
\begin{aligned}
& a_{3 r}:=q^{-6 r^{2}+r}, \\
& a_{3 r+1}:=-q^{-6 r^{2}-r}, \\
& a_{3 r+2}:=q^{-6 r^{2}-5 r-1}-q^{-6 r^{2}-7 r-2} .
\end{aligned}
$$

Therefore for $k>0$ we have

$$
A_{k}+A_{-k}=2 A_{0}+2 \frac{J_{2}^{2}}{J_{1}} \sum_{r=1}^{k}(-1)^{r} q^{-r(r-1) / 2}-J_{1} \sum_{r=1}^{k} a_{r} .
$$

We calculate the following sum two ways. By using 1.10 we have

$$
\begin{aligned}
& \sum_{k=-\infty}^{\infty}(-1)^{k} A_{k} q^{k^{2}}=\sum_{k=-\infty}^{\infty} \sum_{n=1}^{\infty} \sum_{r=1}^{n}(-1)^{r-1} q^{2 n^{2}-n+k^{2}-(r-k)(r-k-1) / 2}\left(1+q^{2 n}\right) \\
& =\sum_{k=-\infty}^{\infty} \sum_{n=1}^{\infty} \sum_{r=1}^{n}(-1)^{r-1} q^{2 n^{2}-n+(k+r)(k+r-1) / 2-r(r-1)}\left(1+q^{2 n}\right) \\
& =\sum_{k=-\infty}^{\infty} q^{k(k-1) / 2} \sum_{n=1}^{\infty} \sum_{r=1}^{n}(-1)^{r-1} q^{2 n^{2}-n-r(r-1)}\left(1+q^{2 n}\right) \\
& =2 \frac{J_{2}^{2}}{J_{1}} \sum_{n=1}^{\infty} \sum_{r=1}^{n}(-1)^{r-1} q^{2 n^{2}-n-r(r-1)}\left(1+q^{2 n}\right) \\
& =2 \frac{J_{2}^{2}}{J_{1}} \sum_{n=-\infty}^{\infty} \sum_{r=1}^{|n|}(-1)^{r-1} q^{2 n^{2}-n-r(r-1)} .
\end{aligned}
$$

This time we let

$$
Q(n, r)=2 n^{2}-n-r(r-1)
$$

and observe that

$$
Q(k-j, k-2 j)=k^{2}-2 j^{2}-j, \quad Q(j-k, k-2 j+1)=k^{2}-2 j^{2}+j .
$$


It follows that

$$
\begin{aligned}
\sum_{n=-\infty}^{\infty} \sum_{r=1}^{|n|}(-1)^{r-1} q^{2 n^{2}-n-r(r-1)} & =\sum_{n=1}^{\infty} \sum_{r=1}^{n}(-1)^{r-1} q^{2 n^{2}-n-r(r-1)}+\sum_{n=-\infty}^{-1} \sum_{r=1}^{-n}(-1)^{r-1} q^{2 n^{2}-n-r(r-1)} \\
& =\sum_{j=0}^{\infty} \sum_{k=2 j+1}^{\infty}(-1)^{k+1} q^{k^{2}-2 j^{2}-j}+\sum_{j=1}^{\infty} \sum_{k=2 j}^{\infty}(-1)^{k} q^{k^{2}-2 j^{2}+j} \\
& =\sum_{m=1}^{\infty} \sum_{k=m}^{\infty}(-1)^{m+k} q^{k^{2}-m(m-1) / 2}=\sum_{k=1}^{\infty} \sum_{j=1}^{k}(-1)^{j+k} q^{k^{2}-j(j-1) / 2}
\end{aligned}
$$

and from 4.45 we have

$$
\sum_{k=-\infty}^{\infty}(-1)^{k} A_{k} q^{k^{2}}=2 \frac{J_{2}^{2}}{J_{1}} \sum_{k=1}^{\infty} \sum_{j=1}^{k}(-1)^{j+k} q^{k^{2}-j(j-1) / 2}
$$

Next we calculate the sum on the left side of 4.45 using 4.44) and find that

$$
\sum_{k=-\infty}^{\infty}(-1)^{k} A_{k} q^{k^{2}}=\frac{J_{1}^{2}}{J_{2}} A_{0}-J_{1} \sum_{k=1}^{\infty} \sum_{r=1}^{k}(-1)^{k} q^{k^{2}} a_{r}+2 \frac{J_{2}^{2}}{J_{1}} \sum_{k=1}^{\infty} \sum_{r=1}^{k}(-1)^{r+k} q^{k^{2}-r(r-1) / 2} .
$$

By 4.45 and 4.46) we have

$$
\frac{J_{1}^{2}}{J_{2}} A_{0}=J_{1} \sum_{k=1}^{\infty} \sum_{r=1}^{k}(-1)^{k} q^{k^{2}} a_{r}
$$

Next we proceed as in the proof of Lemma 4.9 and define analogous functions $G(r, k):=$ $(-1)^{k} q^{k^{2}} a_{r}$ and $F(m, n):=\operatorname{sg}(m)(-1)^{n-1} q^{n(3 n-1)-2 m^{2}+m}$. The analogs of equations 4.15)4.17) hold and we have

$$
\begin{aligned}
& \frac{J_{1}^{2}}{J_{2}} U(q)=\sum_{k=1}^{\infty} \sum_{r=1}^{k}(-1)^{k} q^{k^{2}} a_{r}=\sum_{k=1}^{\infty} \sum_{r=1}^{k} G(r, k)=\sum_{n=-\infty}^{\infty} \sum_{m=1-|n|}^{|n|} F(m, n) \\
& =\sum_{n=1}^{\infty} \sum_{m=1-n}^{n} \operatorname{sg}(m)(-1)^{n-1} q^{n(3 n-1)-2 m^{2}+m}\left(1+q^{2 n}\right) .
\end{aligned}
$$

We are now ready to complete the proof of Conjecture 1.1 .

Proof of congruence (1.2) in Conjecture 1.1. We need a result similar to Lemma 4.5. We define

$$
\epsilon_{1}(m, n)=\frac{1}{2}\left(\operatorname{sg}(m)(-1)^{n-1}-\operatorname{sg}(n)(-1)^{m-1}\right) .
$$


We note that $\epsilon_{1}(m, n)$ only takes the values $0, \pm 1$. Then by 4.43 and 4.9 we have

$$
U(q)-\psi(q) \equiv 2 D_{u}(q) \quad(\bmod 4)
$$

where

$$
D_{u}(q):=\sum_{n=1}^{\infty} d_{u}(n) q^{n}:=\sum_{n=-\infty}^{\infty} \sum_{1-|n| \leq m \leq|n|} \epsilon_{1}(m, n) q^{n(3 n-1)-m(2 m-1)} .
$$

The proof of 4.49 is completely analogous to that of Lemma 4.5 .

We assume $\ell \equiv 7,11,13,17(\bmod 24)$ is prime and let $n, k$ be integers where $\left(\frac{k}{\ell}\right)=-1$. We suppose that

$$
m=\ell^{2} n+k l-s(\ell)
$$

and recall that $s(\ell)=\frac{1}{24}\left(\ell^{2}-1\right)$. Then

$$
24 m-1=\ell(\ell(24 n-1)+24 k) \equiv 24 k \ell \quad\left(\bmod \ell^{2}\right) .
$$

Hence $\ell \| 24 m-1$ and $d_{0}(m)=0$ by Corollary 4.7 , since $\ell \equiv \pm 7, \pm 11(\bmod 24)$. We note that

$$
k \ell-s(\ell) \equiv(24 k \ell+1) \delta_{\ell} \quad\left(\bmod \ell^{2}\right)
$$

Therefore

$$
N_{\psi}(m)=N_{\psi}\left(\ell^{2} n+k \ell-s(\ell)\right) \equiv 0 \quad(\bmod 4)
$$

by Theorem 4.14 since $\ell \equiv \pm 7, \pm 11(\bmod 24)$ and

$$
\left(\frac{24 k}{\ell}\right)=\left(\frac{6}{\ell}\right)\left(\frac{k}{\ell}\right)=1=\varepsilon(\ell) .
$$

Since $d_{0}(m)=0$ we have $d_{u}(m)=0$ and by 4.49 we have

$$
u(m) \equiv N_{\psi}(m)+2 d_{u}(m) \equiv N_{\psi}(m) \quad(\bmod 4),
$$

and

$$
u\left(\ell^{2} n+k l-s(\ell)\right)=u(m) \equiv 0 \quad(\bmod 4)
$$

\section{Conclusion}

The main goal of this paper was to prove the mod 4 unimodal sequence conjectures of Bryson, Ono, Pitman and Rhoades [18] and Kim, Lim and Lovejoy [31. We also proved a related mod 4 conjecture for the Andrews spt-function. The crucial part of the proofs was the connection with the Hurwitz class number. Along the way we needed to study the mod 4 behaviour of the coefficients of certain mock theta functions. As mentioned before, the parity of these was determined very recently by Wang [42. It would be interesting to determine whether the methods of this paper can be used to extend Wang's parity results to mod 4 results for other mock theta functions. 
In this paper we have found a number new Hecke-Rogers identities (4.9), (4.43) and (4.41) altuhec. Can these identities be proved using the Bailey pair machinery [4, Ch.3]? What are the missing Bailey pairs?

Acknowledgments. We would like to thank Jonathan Bradley-Thrush, Chris JenningsShaffer, Jeremy Lovejoy and Eric Mortenson for their comments and suggestions.

\section{REFERENCES}

[1] Scott Ahlgren, Kathrin Bringmann, and Jeremy Lovejoy, $\ell$-adic properties of smallest parts functions, Adv. Math. 228 (2011), no. 1, 629-645. MR2822242

[2] George E. Andrews, The number of smallest parts in the partitions of n, J. Reine Angew. Math. 624 (2008), 133-142. MR2456627

[3] George E. Andrews, Applications of basic hypergeometric functions, SIAM Rev. 16 (1974), 441-484. MR352557

[4] George E. Andrews, q-series: their development and application in analysis, number theory, combinatorics, physics, and computer algebra, CBMS Regional Conference Series in Mathematics, vol. 66, Published for the Conference Board of the Mathematical Sciences, Washington, DC; by the American Mathematical Society, Providence, RI, 1986. MR858826

[5] George E. Andrews, q-orthogonal polynomials, Rogers-Ramanujan identities, and mock theta functions, Tr. Mat. Inst. Steklova 276 (2012), no. Teoriya Chisel, Algebra i Analiz, 27-38. MR2986107

[6] George E. Andrews and Bruce C. Berndt, Ramanujan's lost notebook. Part I, Springer, New York, 2005. MR2135178

[7] George E. Andrews, Freeman J. Dyson, and Dean Hickerson, Partitions and indefinite quadratic forms, Invent. Math. 91 (1988), no. 3, 391-407. MR928489

[8] George E. Andrews, Frank G. Garvan, and Jie Liang, Self-conjugate vector partitions and the parity of the spt-function, Acta Arith. 158 (2013), no. 3, 199-218. MR3040662

[9] George E. Andrews and Dean Hickerson, Ramanujan's "lost" notebook. VII. The sixth order mock theta functions, Adv. Math. 89 (1991), no. 1, 60-105. MR1123099

[10] George E. Andrews, Donny Passary, James A. Sellers, and Ae Ja Yee, Congruences related to the Ramanujan/Watson mock theta functions $\omega(q)$ and $\nu(q)$, Ramanujan J. 43 (2017), no. 2, 347-357. MR3649013

[11] A. O. L. Atkin, Multiplicative congruence properties and density problems for $p(n)$, Proc. London Math. Soc. (3) 18 (1968), 563-576. MR227105

[12] A. O. L. Atkin and P. Swinnerton-Dyer, Some properties of partitions, Proc. London Math. Soc. (3) 4 (1954), 84-106. MR0060535

[13] Bruce C. Berndt and Song Heng Chan, Sixth order mock theta functions, Adv. Math. 216 (2007), no. 2, 771-786. MR2351377

[14] Olivia Beckwith, Martin Raum, and Olav K. Richter, Non-holomorphic ramanujan-type congruences for hurwitz class numbers, arXiv preprint arXiv:2004.09886 (2020).

[15] A. I. Borevich and I. R. Shafarevich, Number theory, Translated from the Russian by Newcomb Greenleaf. Pure and Applied Mathematics, Vol. 20, Academic Press, New York-London, 1966. MR0195803

[16] Kathrin Bringmann, On the explicit construction of higher deformations of partition statistics, Duke Math. J. 144 (2008), no. 2, 195-233. MR2437679

[17] Kathrin Bringmann and Ben Kane, Class numbers and representations by ternary quadratic forms with congruence conditions, arXiv preprint arXiv:2002.01835 (2020).

[18] Jennifer Bryson, Ken Ono, Sarah Pitman, and Robert C. Rhoades, Unimodal sequences and quantum and mock modular forms, Proc. Natl. Acad. Sci. USA 109 (2012), no. 40, 16063-16067. MR2994899 
[19] J. G. Bradley-Thrush, Properties of the Appell-Lerch function (I), Ramanujan J., to appear.

[20] R. Chen and F. G. Garvan., Congruences modulo 4 for weight 3/2 eta-products, Bull. Austral. Math. Soc., doi:10.1017/S0004972720000982, to appear.

[21] Dandan Chen and Liuquan Wang, Representations of mock theta functions, Adv. Math. 365 (2020), 107037. MR4064766

[22] Henri Cohen, A course in computational algebraic number theory, Graduate Texts in Mathematics, vol. 138, Springer-Verlag, Berlin, 1993. MR1228206

[23] David A. Cox, Primes of the form $x^{2}+n y^{2}$, A Wiley-Interscience Publication, John Wiley \& Sons, Inc., New York, 1989, Fermat, class field theory and complex multiplication. MR1028322

[24] F. G. Garvan, Congruences for Andrews'spt-function modulo 32760 and extension of Atkin's Hecke-type partition congruences, Number theory and related fields, Springer Proc. Math. Stat., vol. 43, Springer, New York, 2013, pp. 165-185. MR3081040

[25] F. G. Garvan, Universal mock theta functions and two-variable Hecke-Rogers identities, Ramanujan J. 36 (2015), no. 1-2, 267-296. MR3296723

[26] Basil Gordon and Richard J. McIntosh, Some eighth order mock theta functions, J. London Math. Soc. (2) 62 (2000), no. 2, 321-335. MR1783627

[27] H. Hasse, Über die Teilbarkeit durch $2^{3}$ der Klassenzahl der quadratischen Zahlkörper mit genau zwei verschiedenen Diskriminantenprimteilern, Math. Nachr. 46 (1970), 61-70. MR281699

[28] Kazuhiro Hikami and Jeremy Lovejoy, Torus knots and quantum modular forms, Res. Math. Sci. 2 (2015), Art. 2, 15. MR3324400

[29] Dean R. Hickerson and Eric T. Mortenson, Hecke-type double sums, Appell-Lerch sums, and mock theta functions, I, Proc. Lond. Math. Soc. (3) 109 (2014), no. 2, 382-422. MR3254929

[30] G. Humbert, Formules relatives aux nombres de classes des formes quadratiques binaires et positives, Journ. de Math. (6) 3 (1907), 337-449 (French).

[31] Byungchan Kim, Subong Lim, and Jeremy Lovejoy, Odd-balanced unimodal sequences and related functions: parity, mock modularity and quantum modularity, Proc. Amer. Math. Soc. 144 (2016), no. 9, 3687-3700. MR3513531

[32] Jeremy Lovejoy, Overpartitions and real quadratic fields, J. Number Theory 106 (2004), no. 1, $178-186$. MR2049600

[33] Richard J. McIntosh, Second order mock theta functions, Canad. Math. Bull. 50 (2007), no. 2, $284-290$. MR2317449

[34] Eric T. Mortenson, A Kronecker-type identity and the representations of a number as a sum of three squares, Bull. Lond. Math. Soc. 49 (2017), no. 5, 770-783. MR3742444

[35] Eric Mortenson, On three third order mock theta functions and Hecke-type double sums, Ramanujan J. 30 (2013), no. 2, 279-308. MR3017930

[36] Eric T. Mortenson, On the dual nature of partial theta functions and Appell-Lerch sums, Adv. Math. 264 (2014), 236-260. MR3250284

[37] Arnold Pizer, On the 2-part of the class number of imaginary quadratic number fields, J. Number Theory 8 (1976), no. 2, 184-192. MR406975

[38] Srinivasa Ramanujan, The lost notebook and other unpublished papers, Springer-Verlag, Berlin; Narosa Publishing House, New Delhi, 1988, With an introduction by George E. Andrews. MR947735

[39] Srinivasa Ramanujan, Collected papers of Srinivasa Ramanujan, AMS Chelsea Publishing, Providence, RI, 2000, Edited by G. H. Hardy, P. V. Seshu Aiyar and B. M. Wilson, Third printing of the 1927 original, With a new preface and commentary by Bruce C. Berndt. MR2280843

[40] G. N. Watson, Generating functions of class-numbers, Compositio Math. 1 (1935), 39-68. MR1556875

[41] G. N. Watson, The Final Problem : An Account of the Mock Theta Functions, J. London Math. Soc. 11 (1936), no. 1, 55-80. MR1573993

[42] Liuquan Wang, Parity of coefficients of mock theta functions, arXiv preprint arXiv:2007.03664 (2020). 
[43] Brandon Williams, Vector-valued Hirzebruch-Zagier series and class number sums, Res. Math. Sci. 5 (2018), no. 2, Paper No. 25, 13. MR3799151

[44] Don Zagier, Vassiliev invariants and a strange identity related to the Dedekind eta-function, Topology 40 (2001), no. 5, 945-960. MR1860536

School of Mathematical Sciences, East China Normal University, Shanghai, People's Republic of ChinA

Email address: rchen@stu.ecnu.edu.cn

Department of Mathematics, University of Florida, Gainesville, FL 32611-8105

Email address: fgarvan@ufl.edu 